\title{
A Correlation Between Circumstellar Disks and Rotation in the Upper Scorpius OB Association
}

\author{
S. E. Dahm ${ }^{1}$, Catherine L. Slesnick ${ }^{2,3}, \&$ R. J. White ${ }^{4}$
}

\begin{abstract}
We present projected rotational velocities for 20 early-type (B8-A9) and 74 late-type (F2-M8) members of the $\sim 5$ Myr old Upper Scorpius OB Association derived from high dispersion optical spectra obtained with the High Resolution Echelle Spectrometer (HIRES) on Keck I and the Magellan Inamori Kyocera Echelle (MIKE) on the Magellan Clay telescope. The spectroscopic sample is composed of stars and brown dwarfs with infrared signatures of circumstellar disks, both primordial and debris, and non-excess sources of comparable spectral type. We merge projected rotational velocities, accretion diagnostics, and Spitzer Space Telescope Infrared Array Camera (IRAC) and Multiband Imaging Photometer for Spitzer (MIPS) $24 \mu \mathrm{m}$ photometry to examine the relationship between rotation and circumstellar disks. The rotational velocities are strongly correlated with spectral type, a proxy for mass, such that the median $v \sin i$ for B8-A9 type stars is: $195 \pm 70 \mathrm{~km} \mathrm{~s}^{-1}, \mathrm{~F} 2-\mathrm{K} 4: 37.8 \pm 7.4 \mathrm{~km} \mathrm{~s}^{-1}, \mathrm{~K} 5-\mathrm{K} 9: 13.8_{-8.2}^{+21.3}$ $\mathrm{km} \mathrm{s}^{-1}, \mathrm{M} 0-\mathrm{M} 5: 16.52 \pm 5.3 \mathrm{~km} \mathrm{~s}^{-1}$, and M5.5-M8: $17.72 \pm 8.1 \mathrm{~km} \mathrm{~s}^{-1}$. We find with a probability of $\geq 0.99$ that M-type stars and brown dwarfs having infrared excess suggestive of circumstellar disks rotate more slowly than their non-excess counterparts. A similar correlation is present among F2-K9 type stars, but only at the $\sim 97 \%$ confidence level. Among the early-type (B8-A9) members, rotational velocities of the debris-disk and non-disk populations are indistinguishable. Considering the late-type (F2-M8) stars and brown dwarfs, we find a low fraction of slowly rotating, non-excess sources relative to younger star forming regions, suggesting that most have spun up following disk dissipation. The few late-type (F2-M5) debris disk sources, which may be representative of stars that have recently dispersed their inner disks, are evenly divided between slow and moderate rotators.
\end{abstract}

\footnotetext{
${ }^{1}$ W. M. Keck Observatory, 65-1120 Mamalahoa Hwy, Kamuela, HI 96743

${ }^{2}$ Department of Terrestrial Magnetism, Carnegie Institution for Science, Washington, DC 20015

${ }^{3}$ Current address: Charles Stark Draper Laboratory, 555 Technology Sq MS 31, Cambridge MA 02139

${ }^{4}$ Department of Physics and Astronomy, Georgia State University, Atlanta, GA 30303, USA
} 
Subject headings: clusters: individual (Upper Scorpius OB Association) — stars: pre-main sequence — stars: formation — accretion, accretion disks — stars: rotation

\section{Introduction}

Understanding the evolution of angular momentum in the star formation process remains a fundamental theoretical problem despite decades of effort directed toward its resolution. Simply stated, the specific angular momentum of a typical molecular cloud core is several orders of magnitude greater than that of a solar-mass, zero-age main sequence (ZAMS) star (for a review see Bodenheimer 1995). While a combination of mechanisms is likely responsible for the angular momentum loss over the entirety of pre-main sequence evolution, the near-ubiquitous presence of circumstellar disks around protostars is suggestive of their critical role in the early regulation of angular momentum.

Stellar rotation rates are generally determined observationally using one of two techniques. Many early studies of rotation in pre-main sequence stars employed high-dispersion optical spectra to measure rotational line broadening (e.g. Vogel \& Kuhi 1981; Hartmann et al. 1986; Bouvier et al. 1986). The resulting projected rotational velocities $(v \sin i)$ are subject to uncertainties in the inclination of the stellar rotational axis relative to the line of sight, but can be determined from single observations and are not impacted by accretion-induced variability. Later investigations directly measured rotation periods of pre-main sequence stars by photometric monitoring (e.g. Herbst et al. 1986; Bouvier et al. 1993; Bouvier et al. 1997; Makidon et al. 2004; Lamm et al. 2004). These time-series observations are sensitive to active regions on the stellar surface and allow for the determination of rotation periods with an accuracy of $\sim 1 \%$. For accreting sources, however, variability complicates the determination of rotation periods, potentially biasing photometric surveys toward non-accreting sources.

Initial studies into the relationship between rotation and circumstellar disks for premain sequence stars found that T Tauri stars (TTS) are in general slow rotators with mean rotational velocities of $\sim 15 \mathrm{~km} \mathrm{~s}^{-1}$ (Bodenheimer 1995 and references therein). The low rotational velocities imply that low-mass stars lose most of the specific angular momentum inherited from their parent molecular cloud well before they become optically detectable (Hartmann et al. 1986). Theoretical arguments given to explain the observed slow rotators generally invoke a manifestation of the disk locking mechanism whereby young pre-main sequence stars transfer angular momentum to their surrounding circumstellar disk (Königl 1991) or to an accretion-driven wind originating at the boundary between the disk and the 
stellar magnetosphere (Shu et al. 2000; Matt \& Pudritz 2005). In these scenarios stellar rotation is regulated by the accretion disk. Therefore, once accretion stops and the star has dissipated its inner disk, it should become 'unlocked' and begin to spin up as it contracts toward the main sequence. Observational evidence in regards to this disk-braking paradigm, however, is conflicting.

Early investigations in favor of the classical disk-braking scenario (e.g. Edwards et al. 1993; Bouvier et al. 1993) that used photometrically derived rotation periods, found that accreting classical TTS (CTTS) have longer rotation periods on average than nonaccreting, weak-line TTS (WTTS) in young ( $\lesssim 3$ Myr) star forming regions. The photometric monitoring campaigns of Attridge \& Herbst (1992), Choi \& Herbst (1996), and Herbst et al. (2002) identified a bimodal period distribution in the $\sim 1$ Myr old Orion Nebula Cluster (ONC), which was interpreted as evidence for disk-braking among the slower rotators with masses $\geq 0.25 \mathrm{M}_{\odot}$. Stassun et al. (1999, 2001), however, challenged these results arguing that rotation periods in the ONC were not significantly different from being uniformly distributed. Lamm et al. (2005) provided additional support for the disk regulation scenario finding that slower rotators are more likely to show evidence of circumstellar disks in the $\sim 1-3$ Myr old cluster NGC 2264. Rebull (2001) and Makidon et al. (2004), however, find no clear correlation between rotation period and infrared excess in the ONC or NGC 2264, respectively.

Rebull et al. (2006) attribute the conflicting results to two critical factors: (1) the use of near-infrared (i.e. $J H K$-band) excesses that are insensitive to disks with inner radii extending beyond the dust sublimation limit, and (2) the need for large (i.e. several hundred stars) sample sizes in order to distinguish between locked and freely spinning sources. To address these problems, Rebull et al. (2006) use Spitzer Space Telescope (Spitzer; Werner et al. 2004) observations of $\sim 500$ sources in the ONC with photometrically-derived rotation periods to probe disk emission at larger orbital radii. The Rebull et al. (2006) study finds that nearly all stars with short periods $(\lesssim 2$ days, corresponding to $<v \sin i>\gtrsim 30 \mathrm{~km} / \mathrm{s}$ for a typical TTS) are diskless, providing the most concrete evidence to date that the disk locking paradigm is correct. Among the stars with long periods, about half are associated with disks. Rebull et al. (2006) conclude that of the stars without disks, those with long periods likely have dissipated their disks more recently than those stars with short periods which, presumably, have already had time to spin-up. If this interpretation is correct, we would expect to see a much smaller fraction of diskless stars rotating slowly in more evolved ( 3-10 Myr-old) clusters and associations for which the majority of stars dissipated their inner primordial disks many megayears ago.

Hartmann (2002) also discusses the need for studies of large clusters of intermediate-age 
$(\sim 3-10 \mathrm{Myr})$ pre-main sequence stars. This work re-examines the magnetospheric diskbraking scenario, arguing that the rate of stellar spin-down is limited by the rate at which angular momentum can be removed from the inner disk by viscous processes or by winds. Hartmann (2002) concludes that further measurements of rotation periods as a function of age are neeeded to test the disk-braking hypothesis.

The Upper Scorpius OB association is a critically important region for studies of disk evolution. At $\sim 145$ pc distant, it is among the nearest OB associations to the Sun (Blaauw 1991; de Zeeuw et al. 1999) and has a well-established age of $\sim 5$ Myr (Preibisch \& Zinnecker 1999; Preibisch et al. 2002), when most ( $80 \%)$ optically thick, primordial disks have dissipated (Haisch et al. 2001; Carpenter et al. 2006; Hernandez et al. 2007; Dahm \& Hillenbrand 2007), but many optically thin debris disks remain. Of particular significance, the age dispersion within Upper Scorpius is estimated to be $\leq 3 \mathrm{Myr}$ (Preibisch et al. 2002; Slesnick et al. 2008). Furthermore, Upper Scorpius contains several hundred spectroscopically confirmed members across a large mass range $\left(\sim 15-0.02 \mathrm{M}_{\odot}\right)$. Carpenter et al. (2006) conducted a Spitzer 4.5-16 $\mu \mathrm{m}$ photometric survey of 218 confirmed association members for infrared excess emission, identifying 35 stars with 8 or $16 \mu \mathrm{m}$ excess. Only $19_{-4}^{+5 \%}$ of $\mathrm{K}+\mathrm{M}$ stars in Upper Scorpius were found with infrared excess emission indicative of primordial disks. The follow-up 24 and $70 \mu \mathrm{m}$ photometric survey of Carpenter et al. (2009) identified 19 debris-like systems in Upper Scorpius that exhibit 24 or $70 \mu \mathrm{m}$ excess, but lack excess emission at shorter wavelengths.

Conceptually, primordial disks retain significant quantities of gas and dust that are the remnants of the star formation process. Included within the primordial disk category are sub-classes including: transition disks (Strom et al. 1989), pre-transitional disks (Espaillat et al. 2007), anemic disks (Lada et al. 2006), and homologously depleted disks (Currie et al. 2009), which are all characterized by reduced levels of emission at wavelengths $\leq 10$ $\mu \mathrm{m}$, but that resemble CTTS at longer wavelengths. The 24 and $70 \mu \mathrm{m}$ excesses of debris disk sources presumably originate from the collision of planetesimals (i.e. second-generation dust), although the possibility that these are the remnants of primordial disks with substantial inner holes cannot be ruled out (Carpenter et al. 2009). The $24 \mu \mathrm{m}$ excesses around the late-type $(\mathrm{K}+\mathrm{M})$ debris disk systems included here appear to be distinct from transitional disk systems (Carpenter et al. 2009).

In this work we present projected rotational velocities for a sample of 94 Upper Scorpius stellar and substellar members drawn from the Spitzer mid-infrared surveys of Carpenter et al. (2006) and Slesnick (2007) and the optical photometric and spectroscopic study of Slesnick et al. (2006; 2008). Given the spatial extent of the Upper Scorpius OB association (>100 square degrees), photometric rotation period surveys are more challenging than for 
compact star forming regions like the ONC, NGC 2264, and IC 348. Adams et al. (1998) provide rotation periods for 3 Upper Scorpius members included here, ScoPMS 17: 2.93 days, ScoPMS 31: 1.32 days, and ScoPMS 45: 5.81 days. More comprehensive rotational period surveys are not available. High dispersion spectra were obtained using the High Resolution Echelle Spectrometer (HIRES) on Keck I and the Magellan Inamori Kyocera Echelle (MIKE) on the Magellan Clay telescope. The spectral types of the sample range from B8 to M8 and are nearly equally represented by disk-bearing and non-excess sources as determined by Spitzer 4.5-24 $\mu \mathrm{m}$ observations.

This paper is organized as follows: In Section 2 we describe the Upper Scorpius spectroscopic sample and discuss the HIRES and MIKE observations and reduction. We then discuss (Section 3) the determination of projected rotational velocities and the accretion diagnostics adopted for this analysis. In Section 4 we examine correlations between $v \sin i$ and the presence of circumstellar disks and accretion. We also compare the Upper Scorpius sample with a survey of Taurus-Auriga members over a similar range of spectral types. We discuss the implications of our findings as they relate to the disk-braking scenario and in Section 5 provide a summary of our conclusions.

\section{Observations}

\subsection{Upper Scorpius Membership Sample}

The Upper Scorpius sample was drawn from the Spitzer 4.5-16 $\mu$ m photometric survey of Carpenter et al. (2006) and the optical imaging and spectroscopic survey of Slesnick et al. (2006; 2008). The Carpenter et al. (2006) membership list was compiled from Hipparcos astrometry for the early-type (B-A) stars (de Zeeuw et al. 1999), color-magnitude diagrams with follow-up Li I $\lambda 6708$ observations for G-M type stars (Preibisch \& Zinnecker 1999; Preibisch et al. 2002), and X-ray detected G-M type stars with Li I $\lambda 6708$ confirmation (Walter et al. 1994; Martín 1998; Preibisch et al. 1998; Kunkel 1999; Köhler et al. 2000). Given that the membership selection criteria were based upon stellar properties (proper motions, X-ray activity, Li I $\lambda 6708$ absorption) unrelated to circumstellar disks, it is believed that the sample is unbiased toward the presence or absence of disks.

The very low-mass stars and brown dwarfs included in the spectroscopic sample were drawn from the deep, wide-field $R$ - and $I$-band imaging survey of Slesnick et al. (2006; 2008), who identified 43 sources that have surface gravity signatures consistent with association membership, including 30 brown dwarfs. Spitzer $4.5-24 \mu \mathrm{m}$ photometry for many of these sources are presented in Slesnick (2007). This sample is also considered to be unbiased 
toward the presence or absence of circumstellar disks.

The final spectroscopic sample is composed of 43 disk-bearing stars identified by Carpenter et al. (2006; 2009) and 37 non-excess members selected on the basis of having closely matched spectral types with the disk-bearing sample. Also included are 3 brown dwarfs (spectral types $\geq$ M5.5) with mid-infrared excess and 4 comparable non-excess sources from Slesnick (2007). Finally 7 late-type stars and brown dwarfs that lack mid-infrared observations, but which were found to have strong $\mathrm{H} \alpha$ emission in low resolution spectroscopic observations (Slesnick et al. 2008) were included. Here we confirm with high resolution spectroscopy that 6 of these objects exhibit $\mathrm{H} \alpha$ emission consistent with accretion (see $\S 3.4$ ). By spectral type, the sample is composed of 20 early-type (B8-A9) stars (10 debris disk systems and 10 non-excess sources), 24 F2-K9 type stars (6 primordial disk systems, 5 debris disk systems, and 13 non-excess stars); and 50 M-type sources (21 primordial disk systems, 4 debris disk systems, 18 non-excess sources, and 7 without Spitzer observations). Provided in Table 1 are details of the spectroscopic sample, organized by spectral type taken from Carpenter et al. (2006) and Slesnick et al. (2006; 2008). Also provided is the Spitzer [4.5], [8.0], and [24.0] photometry of Carpenter et al. (2006), Carpenter et al. (2009), and Slesnick (2007) as well as the disk classification (i.e. primordial, debris, non-excess) for each source.

\subsection{High Resolution Echelle Spectrometer Observations}

The High Resolution Echelle Spectrometer (HIRES) is a grating cross-dispersed spectrograph permanently mounted on the Nasmyth platform of Keck I (Vogt et al. 1994). High dispersion spectra were obtained for 50 Upper Scorpius members on the nights of 2006 June 16, 2007 May 24-25, 2011 March 19, and 2011 April 24. The nights were photometric with seeing conditions varying from $0.6-0.9^{\prime \prime}$. HIRES was configured with the red cross-disperser and collimator in beam for increased sensitivity at longer wavelengths. The B2 and C5 deckers $\left(0.574^{\prime \prime} \times 7.0^{\prime \prime}\right.$ and $\left.1.148^{\prime \prime} \times 7.0^{\prime \prime}\right)$, which have projected slit widths of 2 and 4 pixels, were used to provide spectral resolutions of $\sim 66,000$ and 37,500, respectively. Near complete

spectral coverage from $\sim 4800-9200 \AA$ was achieved, a region that includes many gravity and temperature sensitive photospheric features as well as permitted and forbidden transitions generally associated with accretion or chromospheric activity: H $\beta$, He I $\lambda 5876$, O I $\lambda 6300$, $\mathrm{H} \alpha$, [S II] $\lambda \lambda 6717,6730$, and Ca II $\lambda \lambda 8498,8542$, and 8662. The 3-chip mosaic of MIT-LL CCDs with $15 \mu \mathrm{m}$ pixels was used in low gain mode resulting in readout noise levels of 2.8, 3.1 , and $3.1 \mathrm{e}^{-1}$, for the red, green, and blue detectors, respectively. Internal quartz lamps were used for flat fielding and ThAr lamp spectra were obtained for wavelength calibration. Given the range of apparent magnitudes for the observed sample, integration times varied 
from 60 to $2400 \mathrm{~s}$ with typical signal-to-noise ratios of $\sim 30,50$, and 100 being achieved on the blue, green, and red chips, respectively. Radial velocity and spectral standards were observed for a range of spectral types. The HIRES observations were reduced using the MAuna Kea Echelle Extraction, makee, reduction script written by Tom Barlow. Makee is publically available from a link provided on the HIRES webpage.

\subsection{Magellan Inamori Kyocera Echelle Observations}

The Magellan Inamori Kyocera Echelle (MIKE: Bernstein et al. 2003) is a double echelle spectrograph on the Magellan Clay telescope at Las Campanas Observatory. High dispersion red and blue spectra were obtained for 44 stars and brown dwarfs in Upper Scorpius using MIKE over 4 nights from 2008 May 12-15. The nights were photometric and seeing conditions varied from $\sim 0.5-1.0^{\prime \prime}$. MIKE achieves near-complete spectral coverage from $\sim 3500-9400 \AA$ in two simultaneous observations through red $(\lambda \sim 5000-9400 \AA)$ and blue $(\lambda \sim 3500-5000 \AA)$ cameras. The slit width and binning were varied depending upon the brightness of the source, but the majority of the Upper Scorpius members were observed using the $0.7^{\prime \prime}$ slit and $2 \times 2$ binning, yielding a nominal spectral resolution of $\sim 30,000$. The faintest brown dwarf sources were observed with the $1.0^{\prime \prime}$ slit for a spectral resolution of $\sim 22,000$. Integration times for the observed sample ranged from 30 to $2400 \mathrm{~s}$. Internal quartz lamp and ThAr spectra were obtained throughout the nights for flat-fielding and wavelength calibration. Quartz flats and milky flats were obtained at the beginning of each night. The spectra were reduced and extracted using the MIKE pipeline, written by Dan Kelson with methods described in Kelson (2003). The MIKE pipeline is a stand-alone Python script specifically written to pre-proceses and extract MIKE data.

\section{Analysis and Results}

\subsection{Projected Rotational Velocities}

To derive projected rotational velocities, the HIRES and MIKE echelle spectra were first examined to identify orders and spectral regions that are free of telluric features such as the $\mathrm{O}_{2}$ bands near $\lambda \lambda 6280-6310 \AA, \lambda \lambda 6860-6960 \AA$, and $\lambda \lambda 7580-7700 \AA$, or the water absorption bands between $\lambda \lambda 7150-7300 \AA$. Echelle orders containing strong emission features related to accretion or chromospheric activity (e.g. $\mathrm{H} \beta, \mathrm{H} \alpha,[\mathrm{O} \mathrm{I}] \lambda 6300$ ) were also excluded. The remaining orders were then vetted to ensure that high signal-to-noise levels were achieved and that an abundance of photospheric absorption features were present. The echelle orders 
selected from the HIRES observations included, but were not limited to those spanning spectral ranges from: $\lambda \lambda 5620-5710, \lambda \lambda 5700-5800, \lambda \lambda 5900-6100, \lambda \lambda 6100-6140, \lambda \lambda 6320-6420$, and $\lambda \lambda 6430-6540$. The echelle orders selected from the MIKE spectra included those spanning spectral ranges from: $\lambda \lambda 5640-5805, \lambda \lambda 5940-6111, \lambda \lambda 6045-6220, \lambda \lambda 6155-6335$, and $\lambda \lambda 6270-$ 6450. Bluer orders were included for more luminous sources. The minimum measurable $v \sin i$ scales from the velocity resolution of the observations. Given that line widths essentially add in quadrature, we conservatively estimate the minimum measurable $v \sin i$ to be $\sqrt{2}$ times lower than the velocity resolution of the observation or $\sim 3.2$ and $5.7 \mathrm{~km} \mathrm{~s}^{-1}$ for the two slit widths used for the HIRES spectra and $\sim 7.1$ and $9.6 \mathrm{~km} \mathrm{~s}^{-1}$ for those adopted for the MIKE observations.

The projected rotational velocities for the late-type (F-M) Upper Scorpius members were determined by fitting the target spectra with artificially broadened template spectra of slowly rotating $\left(\leq 3.3 \mathrm{~km} \mathrm{~s}^{-1}\right)$ standard stars of similar spectral type. The sources adopted as templates include: HD100180 (G0) $v \sin i=3.3 \mathrm{~km} \mathrm{~s}^{-1}$ (Valenti \& Fischer 2005), HD 92788 (G5) $v \sin i=0.3 \mathrm{~km} \mathrm{~s}^{-1}$ (Valenti \& Fischer 2005), HD 114386 (K3) $v \sin i=0.6 \mathrm{~km} \mathrm{~s}^{-1}$ (Valenti \& Fischer 2005), GJ 424 (M0) $v \sin i<2.5 \mathrm{~km} \mathrm{~s}^{-1}$ (Browning et al. 2010), Gl 555 (M4) $v \sin i$ $<2.5 \mathrm{~km} \mathrm{~s}^{-1}$ (Browning et al. 2010), Gl 876 (M4) $v \sin i<2.0 \mathrm{~km} \mathrm{~s}^{-1}$ (Delfosse et al. 1998), and Gl 406 (M6.5) vsin $i<2.5 \mathrm{~km} \mathrm{~s}^{-1}$ (Browning et al. 2010). These standards are main sequence stars and as such have higher surface gravities ( $\log g \sim 5$ ) than the typical premain sequence object $(\log g \sim 4)$. The lower densities present in the extended atmospheres of $\mathrm{T}$ Tauri stars will result in narrower photospheric absorption features, but the effects of rotational broadening should dominate over both natural and pressure broadening.

The templates were artificially broadened over a range of $v \sin i$ values from 2 to 72 $\mathrm{km} \mathrm{s}^{-1}$ in steps of $2 \mathrm{~km} \mathrm{~s}^{-1}$. For rapid rotators, the upper limit was increased to 400 $\mathrm{km} \mathrm{s}^{-1}$. For each artificially broadened spectrum, a correlation function was determined between the object and the broadened template. A calibration function was then produced coupling the correlation function widths to the $v \sin i$ values of the broadened templates. Using the calibration function and the known peak width of the original correlation function (i.e. the correlation function between the object spectrum and the unbroadened template), the object's projected rotational velocity was determined for each echelle order. We show in Figure 1 (top panel) an example of the correlation function of an object spectrum and template with the rotationally broadened correlation functions overplotted; (middle panel) the empirical $v \sin i$ as a function of peak width relation; and (bottom panel) the best-fitting rotationally broadened template and observed spectra. The mean $v \sin i$ over the optimal echelle orders was adopted as the $v \sin i$ of the target. The standard deviation of the $v \sin i$ values over the optimal echelle orders serves as the uncertainty of the measurement. 
For the early-type (B8-A9) Upper Scorpius members, $v \sin i$ values were determined using one or two echelle orders given the few photospheric absorption features attributable to metals present in their spectra. For the MIKE observations, the orders containing Mg II $\lambda 4481 \AA$ and Si II $\lambda \lambda 6347,6471 \AA$ were used in the cross-correlation analysis. Due to the presence of a blue blocking filter, the HIRES observations were limited only to the Si II $\lambda \lambda 6347,6471 \AA$ features. To serve as template spectra, solar metallicity Kurucz (1979) models having similar effective temperatures $\left(T_{e f f}\right)$ as the Upper Scorpius sources were generated using the stellar spectral synthesis program SPECTRUM written by R. O. Gray. These models adopt main sequence surface gravities $(\log g=5)$, microturbulence velocities of $2 \mathrm{~km} \mathrm{~s}^{-1}$, limb darkening coefficients of 0.6 , and were created with the spectral resolution of the HIRES and MIKE observations. As with the late-type stars, a calibration function was used to relate the correlation function widths to the $v \sin i$ values of the early-type stars. Uncertainties for these $v \sin i$ values were estimated using models of slightly higher and lower $( \pm 500 \mathrm{~K})$ effective temperature. The dispersion present in the resulting $v \sin i$ values was then adopted as the uncertainty of the measurement.

The derived $v \sin i$ values and their associated errors are provided in Table 1. For comparison, we present in Table $2 v \sin i$ values taken from the literature (e.g. Slettebak 1968; Walter et al. 1994; Sartori et al. 2003; Torres et al. 2006; Rice et al. 2010) for 15 common sources. In general there is relative agreement with published velocities with some exceptions, particularly among the rapidly rotating early-type stars.

\subsection{Radial Velocities and Velocity Dispersion}

The relative radial velocity shift between the object and template spectra was determined by the cross-correlation analysis (e.g. the top panel of Figure 1). The heliocentric radial velocities of the standard stars were taken from the literature (e.g. Valenti \& Fischer 2005; Browning et al. 2010; Nidever et al. 2002; Delfosse et al. 1998). Shown in Figure 2 are the distributions of radial velocities for all stars and brown dwarfs included in the spectroscopic sample, binned by spectral type. The median and median absolute deviation of the entire distribution is $-6.28 \pm 1.72 \mathrm{~km} \mathrm{~s}^{-1}$, consistent with the radial velocity reported by de Zeeuw et al. (1999) for the Upper Scorpius OB association, $-4.6 \mathrm{~km} \mathrm{~s}^{-1}$. There are, however, several outliers, particularly among the early type stars that may be singleline, spectroscopic binaries or possibly non-members. The median radial velocity and the $1 \sigma$ velocity dispersion for each spectral type bin are: B8-A9: $-6.14 \pm 15.67 \mathrm{~km} \mathrm{~s}^{-1}, \mathrm{~F} 2-\mathrm{K} 9$ : $-6.31 \pm 4.61 \mathrm{~km} \mathrm{~s}^{-1}$, and M0-M8: $-6.28 \pm 3.04 \mathrm{~km} \mathrm{~s}^{-1}$. These values are consistent with each other and with the accepted radial velocity of the association from de Zeeuw et al. 
(1999). In Table 2 we provide measured radial velocities for $\sim 17$ sources taken from the literature. The largest disparities are found among the early-type stars, several of which are known spectroscopic binaries compiled by Kouwenhoven et al. (2007) and one, HIP 78207, is a confirmed double-line spectroscopic binary ( $(3.3)$.

\subsection{New and Candidate Spectroscopic Binaries}

The high dispersion spectra revealed 1 early-type and 3 late-type double-line spectroscopic binaries that were previously unknown. The classical Be star and possible debris disk system HIP 78207 exhibits 2 sets of absorption features separated by $\sim 40 \mathrm{~km} \mathrm{~s}^{-1}$. The assumed primary, distinguished by broad He I absorption lines, appears to be rapidly rotating and has a radial velocity more consistent with that of the Upper Scorpius OB association. The secondary component exhibits numerous absorption features attributable to Fe I, Fe II, and Si II and has a measured $v \sin i$ of $\sim 38 \mathrm{~km} \mathrm{~s}^{-1}$. Both $\mathrm{H} \alpha$ and $\mathrm{H} \beta$ exhibit broad emission profiles, consistent with the star's Be classification. The late-type double-line spectroscopic binaries include HIP 79462 (G2), J160341.8-200557 (M2), and J160545.4-202308 (M2). HIP 79462 is a debris disk system that appears to be a near-equal mass binary. The velocity separation of the two components is $\sim 63 \mathrm{~km} \mathrm{~s}^{-1}$ with a systemic velocity of $-4.99 \mathrm{~km} \mathrm{~s}^{-1}$ (assuming equal mass). The non-excess source J160341.8-200557 also appears to be a nearequal mass binary with a velocity separation of $\sim 66 \mathrm{~km} \mathrm{~s}^{-1}$ and a systemic velocity of -3.71 $\mathrm{km} \mathrm{s}^{-1}$ (assuming equal mass). The primary and secondary of the primordial disk source J160545.4-202308 are separated by $\sim 40 \mathrm{~km} \mathrm{~s}^{-1}$, with one component exhibiting narrow lines and the other somewhat broader.

Given the single observation per source, confirmation of single-line spectroscopic binaries is not possible. Spectroscopic binary candidates, however, can be identified by comparing the star's radial velocity to that of the association mean; radial velocity dispersions within star forming regions are typically $\sim 2 \mathrm{~km} \mathrm{~s}^{-1}$ (e.g. Hartmann et al. 1986). Five candidate binaries are noted as having broadened cross-correlation functions or by absorption line profiles that appear to exhibit two minima. The correlation functions of these candidates are not significantly asymmetric, which would suggest that they are spectroscopically resolved. The possible spectroscopic binaries include: HD 142987 (G4), J160823.2-193001 (K9), J155918.4-221042 (M4), SCH162007.6-235915.2 (M6), and SCH1622351.58-231727 (M8). Five other candidate binaries are identified on the basis of radial velocities that are at least $\sim 3 \sigma$ from the mean of the of the association $\left(-6.28 \mathrm{~km} \mathrm{~s}^{-1}\right)$. These candidates include: HIP 79739 (reported as a binary by Kouwenhoven et al. 2007), HIP 79124 (also reported as a binary by Kouwenhoven et al. 2007), HIP 78963, HIP 80130, and HIP 82319. 
Several visual binaries are also noted in Table 1. Some of these have been resolved by high angular resolution imaging techniques: [PZ99]J161411.0-230536 (Metchev \& Hillenbrand 2009), ScoPMS 31 (Köhler et al. 2000), and J160643.8-190805 (Dahm, in preparation). These stars are spatially unresolved in the HIRES spectra and their measured velocities are potentially biased by light from two or more sources. Other stars were noted as visual binaries on the HIRES guide camera images (e.g. J160545.4-202308, J160702.1-201938, J160611.9-193532). Follow-up observations are needed to confirm these as physically associated pairs.

\subsection{Accretion Diagnostics}

In the magnetospheric accretion model, gas from the inner disk rim is channeled along magnetic field lines to an impact point on the stellar photosphere (Valenti et al. 1993; Hartmann et al. 1998; Muzerolle et al. 1998). The infalling gas is inferred from inverse $\mathrm{P}$ Cygni line profiles and broadened emission lines of H I, He I, and Ca II, often exceeding several hundred $\mathrm{km} \mathrm{s}^{-1}$ (Hamann \& Persson 1992; Batalha \& Basri 1993).

In our Upper Scorpius spectroscopic sample only one early-type star exhibits H $\alpha$ emission, the spectroscopic binary HIP 78207 (B8). This source was identified as a classical Be star by Hernandez et al. (2005) based upon its lack of near-infrared excess and the presence of strong $\mathrm{H} \alpha$ emission. Emission in classical Be stars is thought to arise from a gaseous disk that is unrelated to accretion. The traditional interpretation of the Be star phenomenon is that they are slightly evolved and undergoing mass loss. The mid-infrared excess associated with this source may arise from hydrogen emission and not from a debris disk.

The remaining Upper Scorpius sources that exhibit $\mathrm{H} \alpha$ emission are late-type stars and brown dwarfs ( $\mathrm{G}-\mathrm{M}$ spectral types). Traditionally the distinction between CTTS and WTTS was based upon the equivalent width of $\mathrm{H} \alpha$, with the demarcating $\mathrm{W}(\mathrm{H} \alpha)$ value established at $10 \AA$ (for a review see Appenzeller \& Mundt 1989). Clear differences in the processes responsible for emission are recognized for CTTS (accretion) and WTTS (chromospheric activity). Because of the contrast effect (Basri \& Marcy 1995; White \& Basri 2003), W(H $\alpha$ ) is spectral type dependent. Consequently, no unique $\mathrm{W}(\mathrm{H} \alpha)$ value is capable of distinguishing all accreting sources from non-accreting objects. Various spectral type dependent $\mathrm{W}(\mathrm{H} \alpha)$ criteria have been proposed (e.g. Martín 1998; White \& Basri 2003), but the full width of $\mathrm{H} \alpha$ emission line profiles at $10 \%$ of peak flux was demonstrated by White \& Basri (2003) to effectively distinguish between optically-veiled and non-veiled pre-main sequence stars. Full widths of $>270 \mathrm{~km} \mathrm{~s}^{-1}$ were found to imply accretion, independent of spectral type. Jayawardhana et al. (2003) adopt a less conservative $10 \% \mathrm{H} \alpha$ full width of $>200 \mathrm{~km} \mathrm{~s}^{-1}$ to 
indicate accretion among very low mass stars and brown dwarfs (M5-M8 spectral types) in IC 348 and Taurus-Auriga. Natta et al. (2004) conclude that $\mathrm{H} \alpha 10 \%$ full widths of $200 \mathrm{~km}$ $\mathrm{s}^{-1}$ for such sources correspond to mass accretion rates $(\dot{M})$ of $\sim 10^{-11} \mathrm{M}_{\odot} \mathrm{yr}^{-1}$.

We adopt a composite criterion for accretion, requiring full widths of $>270 \mathrm{~km} \mathrm{~s}^{-1}$ for spectral types earlier than M5 and $>200 \mathrm{~km} \mathrm{~s}^{-1}$ for types M5 and later. To determine whether the sources in Upper Scorpius are accreting, we first estimate the continuum level by linearly interpolating between regions on either side of $\mathrm{H} \alpha$ that are free of photospheric absorption features and unaffected by the broadened wings of strong $\mathrm{H} \alpha$ emission profiles. The peak level of $\mathrm{H} \alpha$ emission is then determined relative to the defined continuum level. The limits of the $10 \%$ width of peak emission were then defined and the corresponding velocities calculated, which are provided in Table 1.

Just 18 of the 74 late-type (F2-M8) stars and brown dwarfs in the Upper Scorpius sample meet the modified accretion criterion, including two marginal sources: the K2-type star [PZ99]J160421.7-213028 (H $\alpha$ velocity width $\sim 252 \mathrm{~km} \mathrm{~s}^{-1}$, but exhibits an inverse P Cygni profile characteristic of accretion) and the M4-type star J155729.9-225843 ( 238 $\mathrm{km} \mathrm{s}^{-1}$ ). The G4-type, non-excess star HD 142987 exhibits broad $\mathrm{H} \alpha$ emission (H $\alpha$ velocity width $\sim 600 \mathrm{~km} \mathrm{~s}^{-1}$ ), but is a rapid rotator and suspected spectroscopic binary. Three additional sources that are M5 or later (J160611.9-193532, SCH16200756-23591522, and SCH16235158-23172740) exhibit $\mathrm{H} \alpha$ velocity widths $>200 \mathrm{~km} \mathrm{~s}^{-1}$, but are also rapid rotators. Shown in Figure 3 are the emission line profiles for each of the suspected accretors, arranged by spectral type. By spectral type, 0 of 4 F-type stars, 0 of 6 G-type stars, 3 of 14 K-type stars, 11 of $40 \mathrm{M} 0-\mathrm{M} 5$ stars, and 4 of $10 \mathrm{M} 5.5-\mathrm{M} 8$ sources have $\mathrm{H} \alpha$ velocity widths that are consistent with accretion and not the result of rotational broadening. The steady

increase in accretion fraction with spectral type or mass from zero for $\mathrm{F}$ and G-type stars to $\sim 40 \%$ for the latest spectral types parallels the increase in primordial disk fraction.

\section{Correlations with Rotation}

\subsection{Rotation and Stellar Mass}

The dependence of rotation upon stellar mass is a well-established correlation among normal main sequence stars such that rotational velocities peak near $\sim$ B5 spectral types before declining sharply near mid-F (e.g. Slettebak 1955; Kraft 1967; Tassoul 2000). This decline is attributed to the development of a convection zone, from which a magnetic field can be a generated and coupled with a stellar wind. The wind interaction effectively brakes the star, causing a decline in rotation rate with main sequence age $(\tau)$ such that $v \sin i \propto \tau^{-1 / 2}$ 
(Schatzman 1962; Skumanich 1972). The efficiency of angular momentum loss induced by magnetic activity is mass dependent (Scholz et al. 2007). This is observationally supported by the steady progression of remnant rapid rotators toward later spectral types with age: G-M in the $~ 50$ Myr $\alpha$ Persei cluster, mid-K through M in the 100 Myr old Pleiades, and M-type only in the $\sim 600$ Myr Hyades (Delfosse et al. 1998 and references therein).

To examine the mass-dependence of rotation at $\sim 5 \mathrm{Myr}$, we show in Figure 4 the projected rotational velocities of the Upper Scorpius sample as a function of spectral type, a proxy of stellar mass. At this age, a solar mass star would correspond to a spectral type of K7 using the pre-main sequence models of Baraffe et al. (1998) or Siess et al. (2000). A general decline in rotational velocity is apparent from B8 to mid-K spectral types, but appears to be much shallower than is evident among main sequence stars. Figure 4 suggests that the average $v \sin i$ declines from $\sim 100 \mathrm{~km} \mathrm{~s}^{-1}$ to $\leq 20 \mathrm{~km} \mathrm{~s}^{-1}$ between $\sim \mathrm{F} 2$ and $\mathrm{K} 5$ and remains at this level into the substellar mass regime.

Among early-type (B8-A9) stars, $v \sin i$ spans a broad range of values from $<50$ to $\sim 400$ $\mathrm{km} \mathrm{s}^{-1}$. This is comparable to the distribution of $v \sin i$ values for B0-B9 dwarfs in the $\sim 13$ Myr old galactic clusters h \& $\chi$ Persei (Strom et al. 2005). The $v \sin i$ values for late-type stars in Upper Scorpius are similar to those in Taurus-Auriga and Chamaeleon I $(\sim 11-24$ $\mathrm{km} \mathrm{s}^{-1}$, Nguyen et al. 2009), $\eta$ Cha $\left(\sim 5-20 \mathrm{~km} \mathrm{~s}^{-1}\right)$, TW Hydra $\left(\sim 5-32 \mathrm{~km} \mathrm{~s}^{-1}\right)$, and the $\beta$ Pictoris moving group ( $\sim 5-24 \mathrm{~km} \mathrm{~s}^{-1}$, Scholz et al. 2007). We note the presence of a small number of rapid rotators ( $v \sin i \geq 50 \mathrm{~km} \mathrm{~s}^{-1}$ ) among $\mathrm{K}$ and M-type Upper Scorpius members, including one ultrafast ( $v \sin i \sim 100 \mathrm{~km} \mathrm{~s}^{-1}$ ) rotator, [PZ99]J160042.8-212737 (K7).

Shown in Figure 5 are boxplots of $v \sin i$ for the Upper Scorpius sample grouped into bins for B8-A9, F2-K4, K5-K9, M0-M5, and M5.5-M8 spectral types. The F2-K4 and K5-K9 bins straddle the stellar mass boundary where the sharp decline in rotational velocity occurs among main sequence stars. Spectral types earlier than $\sim K 4$, corresponding to masses of $\sim 1.4 \mathrm{M}_{\odot}$, will not have convective zones when on the main sequence as early F-type stars.

The median $v \sin i$ are: $195( \pm 70), 37.8( \pm 7.4), 13.8(+21.3,-8.2), 16.52( \pm 5.3)$, and $17.72( \pm 8.1) \mathrm{km} \mathrm{s}^{-1}$ for the B8-A9, F2-K4, K5-K9, M0-M5, and M5.5-M8 spectral type bins, respectively. The uncertainties presented are the median absolute deviations. The rotational velocities appear to bottom out between K5 and K9 before rising slightly toward later spectral types. The distribution of rotational velocities for the brown dwarfs is notably distinct from that of M0-M5 type stars, showing a substantial dispersion, possibly the result of unresolved spectroscopic binaries among the rapid rotators. These M5 and later spectral types will evolve into late-M and L-type dwarfs, which in the field are more rapidly rotating than early to mid-M type dwarfs (Reiners \& Basri 2010; Blake et al. 2010). If at this age such sources are already spinning more rapidly, the difference may not be attributable to a 
Skumanich type spin-down mechanism.

Nguyen et al. (2009) find clear differences in the $v \sin i$ distributions of F-K stars (24-26 $\mathrm{km} \mathrm{s}^{-1}$ ) and $\mathrm{M}$ dwarfs $\left(11 \mathrm{~km} \mathrm{~s}^{-1}\right)$ in Taurus-Auriga and Chamaeleon I that are statistically significant. Employing the non-parametric Kolmogorov-Smirnov (KS) test to the $v \sin i$ values of our samples of early-type (B8-A9) and late-type (F2-M8) stars, we find a small $\left(10^{-5}\right)$

probability (as defined by Press et al. 1986) that the two samples are drawn from the same parent population. Between the F2-K9 type stars and M-dwarfs, however, the $v \sin i$ distributions are only marginally distinguishable with a $\sim 10 \%$ probability that the two samples are drawn from the same parent population. One possible explanation for this difference with the results of Nguyen et al. (2009) is that the Taurus and Chamaeleon I samples are strongly weighted toward spectral types earlier than M4. Only 6 of their 144 sources have types later than M4 compared with 28 of 94 sources in the Upper Scorpius sample.

Given the long timescales associated with the $v \sin i \propto \tau^{-1 / 2}$ stellar wind braking mechanism of Skumanich (1972), another process must be responsible for the steady decline of rotation rate with mass observed in pre-main sequence stars. Two prominent explanations include 1) star-disk interactions that regulate stellar rotation and 2) changes in internal stellar structure that enhance magnetic activity (Scholz et al. 2007). Growing evidence suggests that disk dissipation timescales are strongly mass-dependent (e.g. Lada et al. 2006; Carpenter et al. 2006; Dahm \& Hillenbrand 2007; Slesnick 2007), such that late-type stars $(\mathrm{K}+\mathrm{M})$ are capable of retaining their primordial disks for prolonged periods relative to their more massive counterparts. Scholz et al. (2007) suggest that at ages >5 Myr, K-type stars have deeper convection zones than $\mathrm{F}-\mathrm{G}$ type stars, implying that rotational braking becomes more effective for these later spectral types. The M-dwarfs, however, remain fully convective, suggesting that the physical mechanism responsible for generating magnetic fields in these stars must be distinct from an $\alpha-\omega$ type dynamo.

\subsection{Rotation and Infrared Excess}

The correlation between rotation and circumstellar disks in the $\sim 1$ Myr old ONC found by Rebull et al. (2006) predicts that disk-bearing and non-excess stars in more evolved clusters should exhibit significantly different period distributions. Hartmann (2002) suggests that the timescales of disk clearing and subsequent spin-up may be comparable. If stars have recently cleared their inner disks, they may still be rotating slowly as though they were experiencing disk-braking. Rebull et al. (2006) predict that a lower fraction of slowly rotating stars lacking disks should be present in older clusters given that stars released from 
disk locking should have had sufficient time to spin-up.

To search for possible correlations between rotation and infrared excess in Upper Scorpius, we merge the projected rotational velocities presented in $\S 3.1$ with the Spitzer IRAC and MIPS photometry of Carpenter et al. (2006; 2009) and Slesnick (2007). The Spitzer survey of Upper Scorpius includes only IRAC channels 2 and 4, limiting our mid-infrared color selection to [4.5]-[8.0] (Rebull et al. 2006 present [3.6]-[8.0] colors in the ONC IRAC survey). In addition to the Spitzer photometry of the Upper Scorpius members, we also adopt the disk classifications of Carpenter et al. (2006; 2009) and Slesnick (2007). Carpenter et al. (2009) assign a primordial disk classification to $\mathrm{K}+\mathrm{M}$ type stars that exhibit 8 and $16 \mu \mathrm{m}$ excesses. Sources with weak excesses at 16 or $24 \mu \mathrm{m}$ were classified as debris disk sources. Slesnick (2007) defines brown dwarfs with [3.6]-[8.0] color excess emission $>3 \sigma$ above photospheric levels as disk-bearing (assumed here to be primordial) sources. We include both primordial and debris disk systems in the disk-bearing sample given that the interiors of debris disks may have only recently dissipated. We also exclude the few doubleline spectroscopic binaries from the statistical analysis. In Figures 6a) and 6b) we plot $v \sin i$ as a function of $[4.5]-[8.0]$ and [4.5]-[24.0] colors, respectively, for the spectroscopic sample. To minimize mass-dependent rotation effects, we divide the sample into 3 spectral type bins: B8-A9, F2-K9, and M0-M8. To facilitate comparison with rotational period surveys (e.g. Rebull et al. 2006), we plot along the right ordinate the rotation periods associated with specific rotational velocities estimated by assuming a stellar radius typical of the spectral type range: $\mathrm{B} 8-\mathrm{A} 9$ stars $2.5 \mathrm{R}_{\odot} ; \mathrm{F} 2-\mathrm{K} 9$ stars $2.0 \mathrm{R}_{\odot}$ (from the pre-main sequence models of Baraffe et al. 1998); and M0-M8 sources $0.88 \mathrm{R}_{\odot}$ (from the same models).

For the early-type stars (B8-A9), we use the KS-test to demonstrate that the diskbearing and non-excess sources are statistically indistinguishable, i.e. the presence or absence of circumstellar disks has no bearing on the observed $v \sin i$ values. These early-type excess sources, however, are associated with debris disks having substantial ( $\geq 10$ AU) inner disk gaps (Carpenter et al. 2006, Dahm \& Carpenter 2009) and may be second-generation debris disks in which dust has been re-generated through the collision of planetesimals.

Among the late-type sources, the distributions of disk-bearing stars and brown dwarfs appear to be shifted toward lower $v \sin i$ than their non-excess counterparts. Performing the KS-test on the $v \sin i$ distributions of disk-bearing and non-excess F2-K9 type stars, we find differences that are significant, but only at the $97.2 \%$ confidence level. The median $v \sin i$ for the disk-bearing and non-excess F2-K9 type stars are 15.4 and $37.8 \mathrm{~km} \mathrm{~s}^{-1}$, respectively. For the M-dwarfs, however, the probability that the $v \sin i$ distributions of the disk-bearing and non-excess sources are drawn from the same parent population is extremely low, $\sim 0.03 \%$, implying a statistically significant difference. This argues in favor of a correlation between 
rotation and circumstellar disks such that disk-bearing sources are rotating more slowly than their non-excess counterparts. The median $v \sin i$ for the disk-bearing and non-excess M-type stars and brown dwarfs are 13.1 and $26.2 \mathrm{~km} \mathrm{~s}^{-1}$, respectively.

While conflicting with the findings of Nguyen et al. (2009) in Taurus-Auriga and Chamaeleon I, this result agrees with those of Rebull et al. (2006) for Orion. The discrepancy with Nguyen et al. (2009) may arise from the substantially lower mass sample included in Upper Scorpius. Shown in Figure 7 are the cumulative distribution functions (CDF) of $v \sin i$ values for the B8-A9, F2-K9, and M0-M8 stars and brown dwarfs with and without mid-infrared excess, determined using the Astronomy Survival (ASURV) analysis package of Feigelson et al. (1996). The CDFs of disk-bearing and non-excess sources of F2-K9 type stars and the M-dwarfs are substantially different. Figure 8 demonstrates this further with box and whisker plots of the $v \sin i$ distributions for disk-bearing and non-excess sources, ordered by spectral type. The boxes for the disk-bearing F2-K9 and M0-M8 sources are clearly displaced relative to those of the non-excess sources.

There are no locking mechanisms available for debris disk systems, but if recently released from their inner disks, such stars could still be experiencing the effects of disk braking. The few late-type debris disks in the sample are nearly evenly divided among slow rotators $\left(v \sin i \sim 10 \mathrm{~km} \mathrm{~s}^{-1}\right)$ and moderate rotators $\left(v \sin i \sim 20 \mathrm{~km} \mathrm{~s}^{-1}\right)$. The single exception is the F2-type debris disk source HIP $79643\left(v \sin i \sim 80 \mathrm{~km} \mathrm{~s}^{-1}\right)$, which more closely resembles the early-type $(\mathrm{B}+\mathrm{A})$ stars. As a whole, the rotational velocity distribution of late-type debris disk systems cannot be distinguished from that of primordial disk-bearing sources, however, the full sample of debris-like systems identified by Carpenter et al. (2009) should be included before more definitive statements are made. There also appears to be few slow rotators among the non-excess sources (\$4.4), which supports the prediction of Rebull et al. (2006) that a lower fraction of long period, non-excess sources should be present at more advanced ages.

\subsection{Rotation and Accretion}

Accretion is generally associated with near- and mid-infrared excess emission originating from hot dust in the inner disk. For 63 of 67 (94\%) pre-main sequence stars in Taurus-Auriga and Chamaeleon I, Nguyen et al. (2009) find that the presence or absence of accretion as determined by the $\mathrm{H} \alpha 10 \%$ velocity width criterion of Jayawardhana et al. (2003) is strongly correlated with Spitzer $8.0 \mu \mathrm{m}$ excess. Shown in Figure 9 are the [4.5]-[8.0] colors for the Upper Scorpius sample plotted as a function of $\mathrm{H} \alpha$ equivalent width, $\mathrm{W}(\mathrm{H} \alpha)$, and $\mathrm{H} \alpha 10 \%$ velocity width for G4-M4 type stars (upper panels) and M5-M8 type sources (lower panels). 
Several non-excess sources fall between the 200 and $270 \mathrm{~km} \mathrm{~s}^{-1}$ boundaries, presumably a result of rotational broadening. A substantial fraction (14 of 26) of primordial disk sources do not appear to be accreting.

We next examine the $v \sin i$ distributions for correlations between accretion and rotation. Only stars hosting gas-rich primordial disks with magnetospheric footprints in the inner disk should experience rotational braking. Considering the late-type (G4-M8) Upper Scorpius members with $\mathrm{H} \alpha$ in emission, we plot in Figure $10 v \sin i$ as a function of $\mathrm{H} \alpha 10 \%$ velocity width. There are too few (3) suspected accretors among the G4-K9 type stars to perform proper statistical analysis. Applying the KS-test to the M0-M8 accreting and non-accreting populations, we find that while the maximum deviation between the two cumulative distribution functions is substantial, the significance level remains low, such that the null hypothesis that accreting and non-accreting sources are drawn from the same parent population cannot be rejected with any degree of confidence.

Nguyen et al. (2009) also find negative KS-test results between accretion and rotation in Taurus-Auriga and Chamaeleon I, which appear to stem from the presence of a large number of rapidly rotating accretors having $v \sin i>20 \mathrm{~km} \mathrm{~s}^{-1}$. Such objects are rare in Upper Scorpius with only one accretor having a comparable rotational velocity. To explain the lack of correlation between accretion and mid-infrared excess and between rotation and accretion in the Upper Scorpius sample, we postulate that some primordial disk-bearing sources may be accreting at levels below the detection threshold of the $\mathrm{H} \alpha$ velocity width criterion. Dahm \& Carpenter (2009) and Dahm (2010) find that many of the primordial disk-bearing stars in Upper Scorpius exhibit reduced levels of near- and mid-infrared excess emission and order of magnitude lower mass-accretion rates compared with comparable Class II sources in Taurus-Auriga. They suggest that the inner disk radii for some of the Upper Scorpius sources exceed their respective dust sublimation radii, potential evidence for inner disk evolution. Such transition-like objects may either be weakly accreting or no longer accreting through the inferred inner disk gap.

\subsection{Implications for Disk Braking}

In the disk braking model, rapidly rotating protostars spin down during the T Tauri phase over the disk braking timescale $\left(\tau_{D B}\right)$. Ignoring spin-up torques induced by accreting material, Hartmann (2002) derives a lower limit for $\tau_{D B}$ given by:

$$
\tau_{D B} \geq 4.5 \times 10^{6} \mathrm{yr}_{0.5} \dot{M}_{-8}^{-1} f
$$

where $\mathrm{M}_{0.5}$ is the stellar mass in units of $0.5 \mathrm{M}_{\odot}, \dot{M}_{-8}$ is the mass accretion rate in units of 
$10^{-8} \mathrm{M}_{\odot} \mathrm{yr}^{-1}$, and $f$ is the ratio of the stellar angular velocity to the breakup velocity. The dependence upon mass accretion rate and the range of $\dot{M}$ values among CTTS of comparable mass and age implies an intrinsic spread in disk braking timescales (Hartmann 2002).

For typical values of $\dot{M}$ and mass in Taurus-Auriga, $\tau_{D B}$ is $\sim 5$ Myr. To explain the lack of correlation between accretion or the presence of inner disks and rotational velocities in Taurus-Auriga and Chamaeleon I, Nguyen et al. (2009) suggest that insufficient time has elapsed for disk braking to take hold in these young systems. This, however, conflicts with the findings of Rebull et al. (2006) in the comparably aged Orion region. Alternatively Nguyen et al. (2009) propose that a substantial age dispersion may be present in these regions that masks the expected signatures of disk braking in the rotational velocity distributions.

The age of Upper Scorpius is approximately equal to the disk braking timescale for a half-solar mass star, a nominal $\dot{M}$ value of $1.0 \times 10^{-9} \mathrm{M}_{\odot} \mathrm{yr}^{-1}$, and $f>0.1$. The age dispersion within Upper Scorpius is estimated to be $\leq 3$ Myr (Slesnick et al. 2008), eliminating this potential source of uncertainty. The correlations between the presence of disks and rotation for M-dwarfs and possibly F-K type stars in Upper Scorpius support the disk braking hypothesis. Circumstantial evidence is provided by the small fraction of non-excess sources that are slow rotators. Shown in the upper panel of Figure 11 are the rotation periods for 464 sources in Orion from Rebull et al. (2006) plotted as a function of [4.5]-[8.0] color. Defining stars having [4.5]-[8.0] colors between -0.2 and +0.2 mag. as non-excess sources, we find that the period distribution for these stars spans from $\leq 1$ to $>25$ days. A substantial fraction $(63 / 140$ or $45 \%)$ of non-excess sources in Orion have rotation periods of $>5$ days. Assuming the Orion sample to be comprised predominantly of late-type stars having radii $\sim 1.5 R_{\odot}$, such periods correspond to equatorial rotation velocities of $\sim 15 \mathrm{~km} \mathrm{~s}^{-1}$ or less.

Shown in the lower panel of Figure 11 are histograms of $v \sin i$ for late-type (F2 and later), non-excess sources in Upper Scorpius as well as Taurus-Auriga and Chamaeleon I (from Nguyen et al. 2009). The fraction of slowly rotating ( $v \sin i \lesssim 15 \mathrm{~km} \mathrm{~s}^{-1}$ ), non-disk bearing sources in Upper Scorpius is found to be 5/30 ( 17\%), while that of Taurus and Chamaeleon I is 23/71 ( $32 \%)$. These first order comparisons with Orion, Taurus-Auriga, and Chamaeleon I are suggestive of a gradual decline in the number of slowly rotating, non-excess sources with age.

Other factors, however, should be considered before attributing these results to disk regulation including: normal accretion-induced variability (e.g. Johns \& Basri 1995), intermittent periods of spin-up or enhanced disk emission from rapid accretion events (see Rebull et al. 2006 and references therein); unrecognized spectroscopic binaries among the rapid rotators; and observational uncertainties including inclination angle. The role of environment in disk evolution and the effect of nearby early-type stars that can ionize and disperse 
molecular gas in the envelopes and disks surrounding low mass populations (e.g. Rebull et al. 2006; O'dell 1998) is poorly understood. The environs of Upper Scorpius is certainly distinct from that found in Taurus-Auriga (a sparsely populated region with few early-type stars) or the ONC (a densely packed cluster with several proximal O-type stars).

\section{Summary and Conclusions}

We have carried out an extensive investigation into the influence of circumstellar disks upon rotation for 94 stars and brown dwarfs in the $\sim 5$ Myr Upper Scorpius OB association using Spitzer IRAC and MIPS $24 \mu \mathrm{m}$ photometry and measured projected rotational velocities from high dispersion spectroscopy. We find that rotational velocity is strongly correlated with spectral type, a proxy for mass, such that the median $v \sin i$ for B8-A9 type stars is: $195 \pm 70 \mathrm{~km} \mathrm{~s}^{-1}$, for F2-K4 types: $37.8 \pm 7.4 \mathrm{~km} \mathrm{~s}^{-1}$, for $\mathrm{K} 5-\mathrm{K} 9: 13.8_{-8.2}^{+21.3} \mathrm{~km} \mathrm{~s}^{-1}$, for M0-M5: $16.52 \pm 5.3 \mathrm{~km} \mathrm{~s}^{-1}$, and for M5.5-M8: $17.72 \pm 8.1 \mathrm{~km} \mathrm{~s}^{-1}$. The distribution of rotational velocities for brown dwarfs (M5.5 and later) exhibits a substantial dispersion that is not present among M0-M5 type stars. This may in part result from unresolved spectroscopic binaries. Late-M and L-type dwarfs in the field are more rapidly rotating than early to mid-M type stars. If this difference is present at $\sim 5 \mathrm{Myr}$, it would suggest that it is not due to a Skumanich type spin-down mechanism.

We find with a probability of $\geq 0.99$ that among M-dwarfs, disk-bearing sources rotate more slowly on average than their non-disk bearing counterparts. Disk-bearing F-K type stars also appear to rotate more slowly than non-excess sources, but only at the $\sim 97 \%$ confidence level. Given that less than half of the F-K type Upper Scorpius members observed by Spitzer have $v \sin i$ measurements available, we suggest that additional high dispersion spectra are needed to adequately explore this mass range and to better quantify this result.

Considering the late-type (F2-M8) stars and brown dwarfs, we find few slowly rotating, non-excess sources in Upper Scorpius, suggesting that most have spun up following disk dissipation. Comparing the rotational velocities of late-type, non-excess sources in Upper Scorpius with those in the presumably younger Taurus-Auriga and Chamaeleon I star forming regions and Orion, we find that the fraction of slow rotators (i.e. $v \sin i \lesssim 15 \mathrm{~km} \mathrm{~s}^{-1}$ ) is lower in Upper Scorpius by a factor of $\sim 2$ or more. This supports the prediction of Rebull et al. (2006) that lower fractions of slowly rotating, non-excess stars should be present in older, more evolved clusters.

Among the small number of late-type (K5-M5), debris disk sources in the Upper Scorpius sample, we find that rotational velocities are nearly evenly divided between slow and 
moderate rotators. Such systems may be representative of second generation debris disks or the remnants of primordial disks with substantial inner holes. These stars may have been recently released from their inner disks and are in the process of spinning up.

The lack of a strong correlation between accretion and rotation among the Upper Scorpius sources possibly originates from the small number of primordial disk systems that are accreting at detectable levels using the $\mathrm{H} \alpha 10 \%$ width accretion criteria of White \& Basri (2003) and Jayawardhana et al. (2003). Alternatively, given the evidence for reduced nearand mid- infrared excess emission among Upper Scorpius primordial disks, it is possible that many inner disks have receded beyond their respective dust sublimation radii and are no longer accreting. Such transition-like objects, if recently released, may still be experiencing the effects of disk braking and may not have had sufficient time to spin up.

The high dispersion spectra have revealed 4 new double-line spectroscopic binaries in Upper Scorpius as well as 5 possible spectroscopic binaries. Additional high resolution spectra are needed for confirmation and to determine the orbital properties of these systems.

Future investigations of rotation and disks should include the remaining debris disk candidates in Upper Scorpius identified by Carpenter et al. (2009) in order to examine whether differences exist between the rotational properties of debris disks and primordial disks. Similar investigations of other young clusters and associations with Spitzer observations available are critically needed over a broad range of ages to confirm the role of circumstellar disks in the regulation of angular momentum in pre-main sequence stars.

This work is based on observations made with the Spitzer Space Telescope, which is operated by the Jet Propulsion Laboratory (JPL), California Institute of Technology, under NASA contract 1407. The Digitized Sky Surveys, which were produced at the Space Telescope Science Institute under U.S. Government grant NAG W-2166, were used as were the the SIMBAD database operated at CDS, Strasbourg, France, and the Two Micron All Sky Survey (2MASS), a joint project of the University of Massachusetts and the Infrared Processing and Analysis Center (IPAC)/California Institute of Technology, funded by NASA and the National Science Foundation. We wish to thank Lynne Hillenbrand and John Carpenter for many insightful discussions and suggestions that greatly improved this manuscript.

\section{REFERENCES}

Adams, N. R., Walter, F. M., and Wolk, S. J. 1998, AJ, 116, 237

Appenzeller, I., \& Mundt, R. 1989, A\&A Rev., 1, 291 
Ardila, D., Martín, E., and Basri, G. 2000, AJ, 120, 479

Attridge, J. M. and Herbst, W. 1992, ApJ, 398, 61

Basri, G. and Marcy, G. W. 1995, AJ, 109, 762

Batalha, C. C. \& Basri, G. 1993, ApJ, 412, 363

Bernstein, R., Shectman, S. A., Gunnels, S. M., Mochnacki, S., and Athey, A. E. 2003, SPIE, 4841, 1694

Blaauw, A. 1991, in The Physics of Star Formation and Early Stellar Evolution, ed. C. J. Lada \& N. D. Kylafis (Dordrecht: Kluwer), 125

Blake, C. H., Charbonneau, D., \& White, R. J. 2010, ApJ, 723, 684

Bodenheimer, P. 1995, ARAA, 33, 199

Bouvier, J., Bertout, C., Benz, W., and Mayor, M. 1986, A\&A, 165, 110

Bouvier, J., Cabrit, S., Fernandez, M., Martín, E., and Matthews, J. M. 1993, A\&A, 272, 176

Bouvier, J. et al. 1997, A\&A, 318, 495

Carpenter, J. M., Mamajek, E. E., Hillenbrand, L. A., \& Meyer, M. R. 2006, ApJ, 651, L49

Carpenter, J. M., Mamajek, E. E., Hillenbrand, L. A., \& Meyer, M. R. 2009, ApJ, 705, 1646

Choi, P. I. and Herbst, W. 1996, AJ, 111, 283

Currie, T., Lada, C. J., Plavchan, P., Robitaille, T. P., Irwin, J., \& Kenyon, S. J. 2009, ApJ, 698,1

de Zeeuw, P. T., Hoogerwerf, R., de Bruijne, J. H. J., Brown, A. G. A., \& Blaauw, A. 1999, AJ, 117,354

Dahm, S. E. and Hillenbrand, L. A. 2007, 133, 2072

Dahm, S. E. and Carpenter, J. M. 2009, AJ, 137, 4024

Dahm, S. E. 2010, AJ, 140, 1444

Edwards, S., Strom, S. E., Hartigan, P., Strom, K. M., Hillenbrand, L. A., Herbst, W., Attridge, J., Merrill, K. M., et al. 1993, AJ, 106, 372

Engelbracht, C. W., Blaylock, M., Su, K. Y. L., Rho, J., Rieke, G. H., Muzerolle, J., Padgett, D. L., Hines, D. C., et al. 2007, PASP, 119, 994

Espaillat, C., Calvet, N., D’Alessio, P., Hernández, J., Qi, C., Hartmann, L., Furlan, E., \& Watson, D. M. 2007, ApJ, 670, L135

Fazio, G. G., Hora, J. L., Allen, L. E., Ashby, M. L. N., Barmby, P., Deutsch, L. K., Huang, J.-S., Kleiner, S. et al. 2004, ApJS, 154, 10 
Guieu, S., Pinte, C., Monin, J.-L., Menard, F., Fukagawa, M., Padgett, D. L., NoriegaCrespo, A., Carey, S. J., et al. 2007, A\&A, 465, 855

Haisch, K. E., Jr., Lada, E. A., \& Lada, C. J. 2001, ApJ, 553, L153

Hartmann, L., Hewett, R., Stahler, S., and Mathieu, R. D. 1986, ApJ, 309, 275

Hamann, F. \& Persson, S. E. 1992, ApJ, 394, 628

Hartmann, L., Calvet, N., Gullbring, E., and D’Alesio, P. 1998, ApJ, 495, 385

Hartmann, L. 2002, ApJ, 566, 29

Hartmann, L, Megeath, S. T., Allen, L., Luhman, K. L., Calvet, N., D’Alessio, P., FrancoHernandez, R., and Fazio, G. 2005, ApJ, 629, 881

Herbst, W., Booth, J. F., Chugainov, P. F., Zajtseva, G. V., Barksdale, W., Covino, E., Terranegra, L., Vittone, A., and Vrba, F. 1986, ApJ, 310, 71

Herbst, W., Bailer-Jones, C. A. L., Mundt, R., Meisenheimer, K., and Wackermann, R., 2002, A\&A, 396, 513

Hernandez, J., Calvet, N., Hartmann, L., Briceno, C., Sicilia-Aguilar, A., and Berlind, P. 2005, AJ, 129, 856

Jayawardhana, R., Mohanty, S., and Basri, G. 2003, ApJ, 592, 282

Johns, C. M., \& Basri, G. 1995, AJ, 109, 2800

Kelson, D. D. 2003, PASP, 115, 688

Koenigl, A. 1991, ApJ, 370, L39

Köhler, R., Kunkel, M., Leinert, C., \& Zinnecker, H. 2000, A\&A, 356, 541

Kouwenhoven, M. B. N., Brown, A. G. A., Portegies, Zwart, S. F., and Kaper, L. 2007, A\&A, 474, 77

Kraft, R. P. 1967, ApJ, 150, 551

Kunkel, M. 1999, Ph.D. thesis, Julius-Maximilians-Univ., Wurzburg

Kurucz, R. L. 1979, ApJSS, 40, 1

Lada, C. J., Muench, A. A., Luhman, K. L., Allen, L., Hartmann, L., Megeath, T., Myers, P. C., Fazio, G., et al., 2006, AJ, 131, 1574

Lamm, M. H., Mundt, R., Bailer-Jones, C. A. L., and Herbst, W. 2005, A\&A, 430, 1005

Luhman, K. L., Lada, C. J., Hartmann, L., Muench, A. A., Megeath, S. T., Allen, L. E., Myers, P. C., Muzerolle, J., et al., 2005, ApJ, 631, 69

Luhman, K. L., Whiteney, B. A., Meade, M. R., Babler, B. L., Indebetouw, R., Bracker, S., and Churchwell, E. B. 2006, ApJ, 647, 1180 
Makidon, R. B., Rebull, L. M., Strom, S. E., Adams, M. T., and Patten, B. M. 2004, AJ, 127,2228

Martín, E. L. 1998, AJ, 115, 351

Martín, E. L., Delfosse, Z., and Guieu, S. 2004, AJ, 127, 449

Natta, A., Testi, L., Muzerolle, J., Randich, S., Comeron, F., and Persi, P. 2004, A\&A, 424, 603

Muzerolle, J., Hartmann, L., \& Calvet, N. 1998, AJ, 116, 455

O'dell, C. R. 1998, AJ, 115, 263

Preibisch, T., Guenther, E., Zinnecker, H., Sterzik, M., Frink, S., \& Roeser, S. 1998, A\&A, 333,619

Preibisch, T., \& Zinnecker, H. 1999, AJ, 117, 2381

Preibisch, T., Brown, A. G. A., Bridges, T., Guenther, E., \& Zinnecker, H. 2002, AJ, 124, 404

Press, W. H., Flannery, B.P., \& Teukolsky, S.A. 1986, Cambridge: University Press

Reach, W. T., Megeath, S. T., Cohen, M., Hora, J., Carey, S., Surace, J., Willner, S. P., Barmby, P., Wilson, G., Glaccum, W., Lowrance, P., Marengo, M., \& Fazio, G. G. 2005, PASP, 117,978

Rebull, L. 2001, AJ, 121, 1676

Rebull, L. M., Stauffer, J. R., Megeath, S. T., Hora, J. L. and Hartmann, L. 2006, ApJ, 646, 297

Reiners, A., \& Basri, G. 2010, ApJ, 710, 924

Riaz, B., Lodieu, N., and Gizis, J. E. 2009, ApJ, 705, 1173

Rieke, G. H., Young, E. T., Engelbracht, C. W., Kelly, D. M., Low, F. J., Haller, E. E., Beeman, J. W., Gordon, K. D., et al. 2004, ApJS, 154, 25

Sartori, M. J., Lepine, J. R. D., and Dias, W. S. 2003, A\&A, 404, 913

Scholz, A., Jayawardhana, R., Wood, K., Meeus, G., Stelzer, B., Walker, C., O'Sullivan, M. 2007, ApJ, 660, 1517

Shu, F. H., Najita, J. R., Shang, H., \& Li, Z.-Y. 2000, Protostars and Planets IV, 789

Skumanich, A. 1972, ApJ, 171, 565

Slesnick, C. L., Carpenter, J. M., \& Hillenbrand, L. A. 2006, AJ, 131, 3016

Slesnick, C. L. 2007, PASP, 119, 1205 
Slesnick, C. L.., Carpenter, J. M., and Hillenbrand, L. A. 2008, ApJ, 688, 377

Stassun, K. G., Mathieu, R. D., Mazeh, T., and Vrba, F. J. 1999, AJ, 194, 7013

Stassun, K. G., Mathieu, R. D., Vrba, F. J., Mazeh, T., and Henden, A. 2001, AJ, 121, 1003

Strom, K. M., Strom, S. E., Edwards, S., Cabrit, S., \& Skrutskie, M. F. 1989, AJ, 97, 1451

Torres, C. A. O., Quast, G. R., da Silva, L., de La Reza, R., Melo, C. H. F., \& Sterzik, M. 2006, A\&A, 460, 695

Vogel, S. N., and Kuhi, L. V. 1981, ApJ, 245, 960

Valenti, J. A., Basri, G., \& Johns, C. M. 1993, AJ, 106, 2024

Vogt, S. S., Allen, S. L., Bigelow, B. C., Bresee, L., Brown, B., Cantrall, T. et al. 1994, SPIE, 2198, 362

Walter, F. M., Vrba, F. J., Mathieu, R. D., Brown, A., \& Myers, P. C. 1994, AJ, 107, 692

White, R. J. \& Basri, G. 2003, ApJ, 582, 1109 

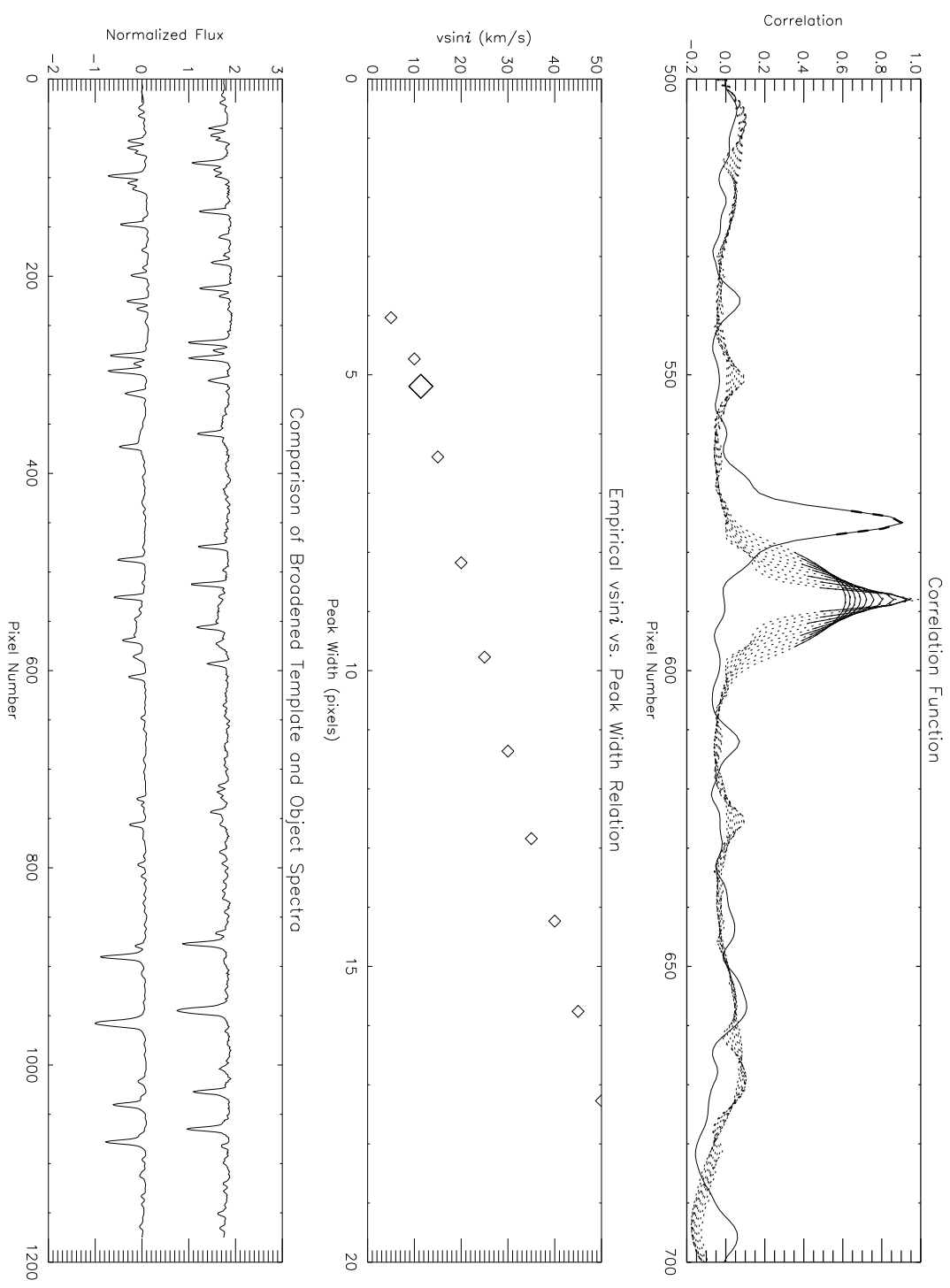

Fig. 1.- (top panel) The correlation function between an object spectrum and a slowly rotating template spectrum with the rotationally broadened correlation functions for 10 velocities between $5 \mathrm{~km} \mathrm{~s}^{-1}$ (narrowest profile) and $50 \mathrm{~km} \mathrm{~s}^{-1}$ (broadest profile) superimposed. The apparent shift between correlation function and the broadened templates is representative of the radial velocity difference between the object and template sources. (middle panel) Empirical $v \sin i$ as a function of peak width of the correlation function. The $v \sin i$ of the best-fitting correlation function is represented by the enlarged symbol. (bottom panel) The best-fitting, rotationally broadened template spectrum and object spectrum. 

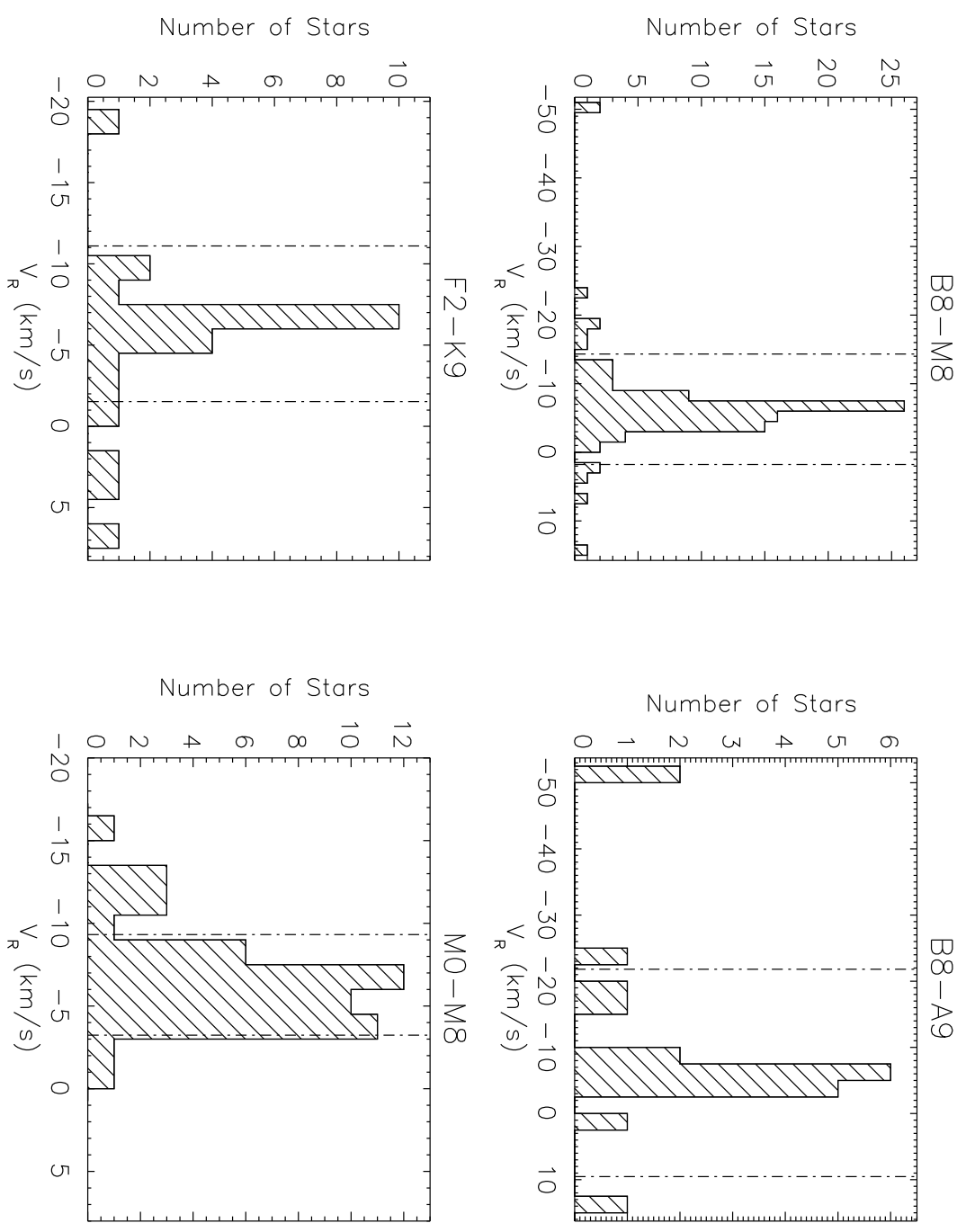

Fig. 2.- The heliocentric radial velocity distribution for all Upper Scorpius members in the spectroscopic sample (upper left panel). The median radial velocity is $-6.15 \mathrm{~km} \mathrm{~s}^{-1}$, consistent with results found in the literature for the Upper Scorpius OB Association. In the other panels are the heliocentric radial velocity distributions by spectral type: B8-A9 (upper right panel), F2-K9 (lower left panel), and M0-M8 (lower right panel). The $1 \sigma$ dispersions are represented by the vertical dot-dashed lines in each panel. The median radial velocities for each spectral type bin are consistent with that for all sources. Several outliers, however, are present, particularly among the early type stars. These may be spectroscopic binaries or non-members. 

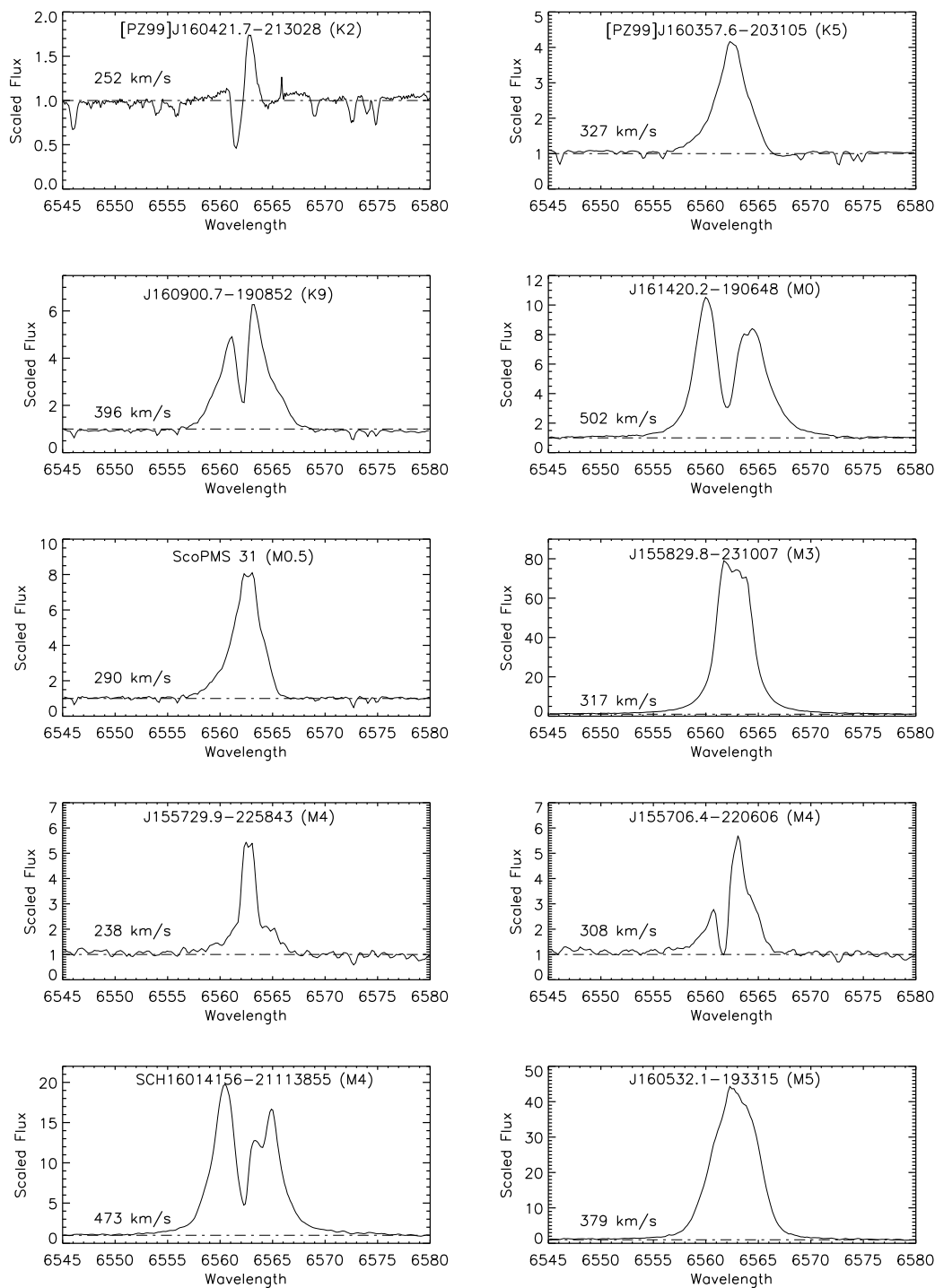

Fig. 3.- $\mathrm{H} \alpha$ emission line profiles for each of the suspected accretors in the Upper Scorpius late-type sample, ordered by spectral type. The assumed continuum level is represented by the dot-dashed line and the measured velocity widths are given in each panel. By spectral type, 0 of 4 F-type stars, 0 of 6 G-type stars, 3 of 14 K-type stars, 11 of 40 M0-M5 stars, and 4 of 10 M5.5-M8 sources have $\mathrm{H} \alpha$ velocity widths consistent with accretion. Two marginal accreting sources are included here for completeness: the K2-type star [PZ99]J160421.7-213028 and the M4-type star J155729.9-225843. 

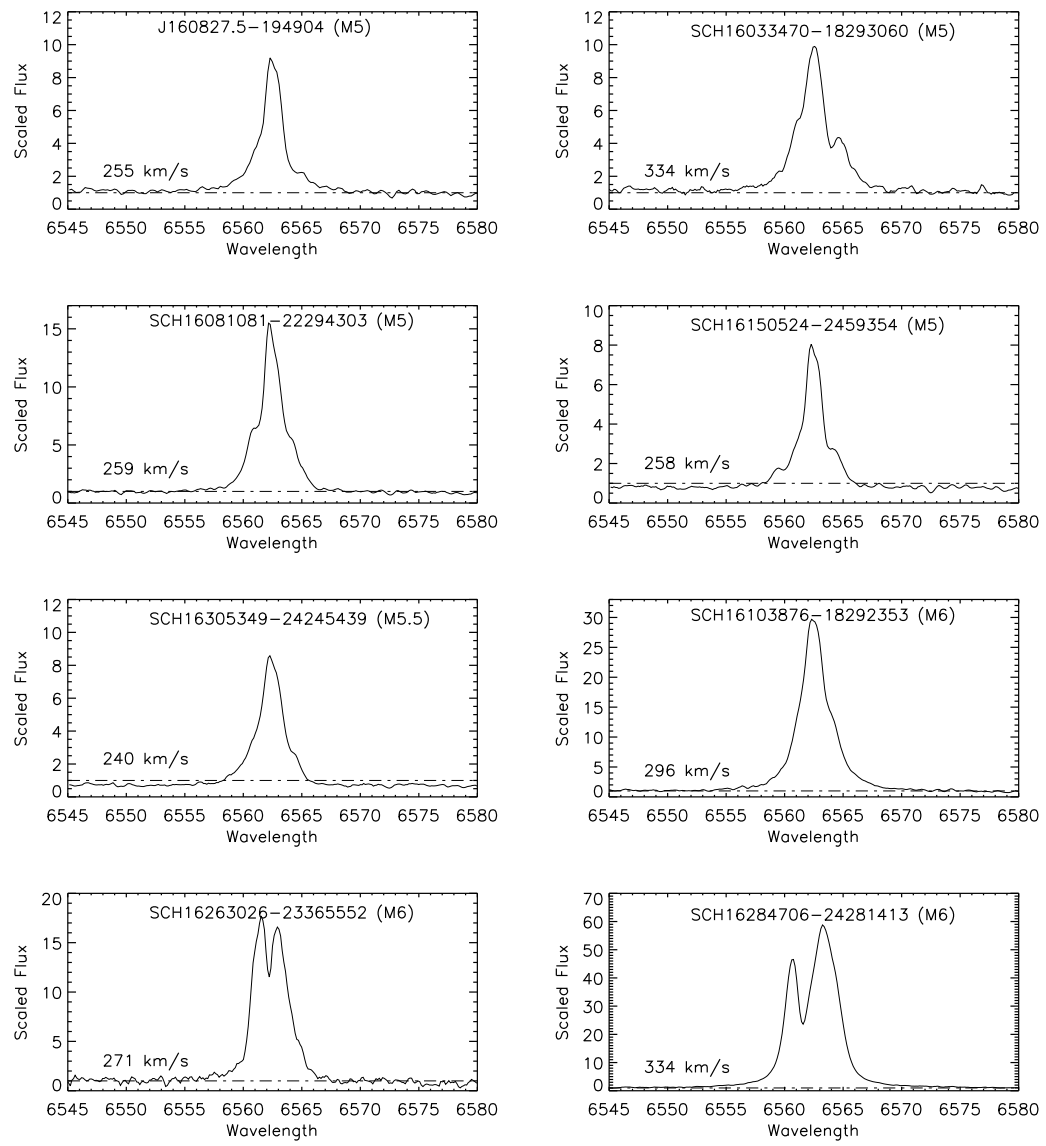

Fig. 3.- (continued) 


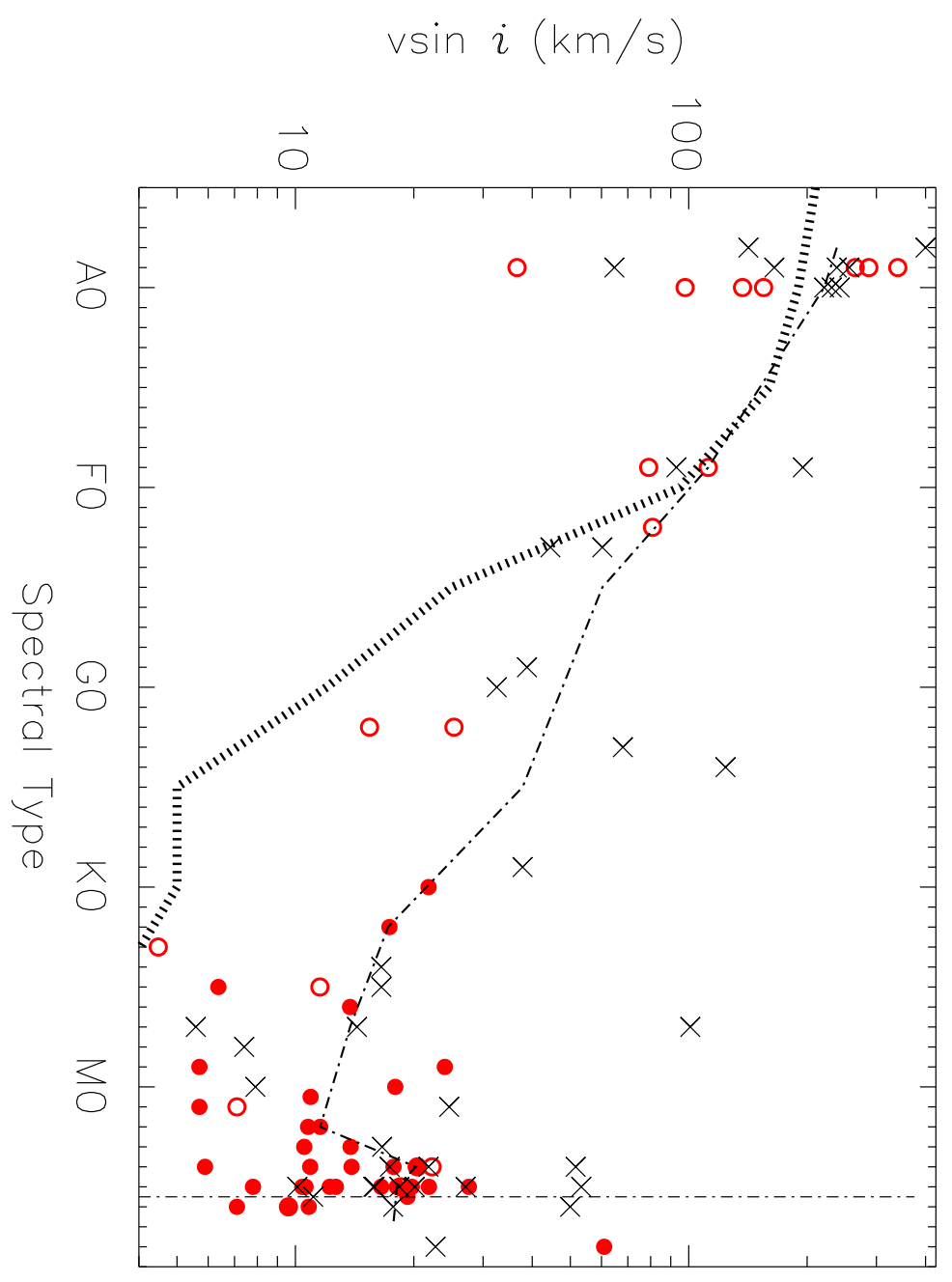

Fig. 4.- Projected rotational velocity as a function of spectral type for the Upper Scorpius sample. Primordial disk-bearing sources are represented by solid red circles, debris disk systems as open red circles, and non-excess sources as crosses. At $\sim 5 \mathrm{Myr}$, a solar mass star would correspond to a $\sim$ K7 spectral type using the pre-main sequence models of Baraffe et al. (1998). The vertical dot-dashed line represents the approximate location of the sub-stellar mass boundary, near M5.5 spectral type at the assumed age of Upper Scorpius. A general decline in rotational velocity is apparent from B8 to early M, paralleling the distribution of main sequence rotational velocities, which are represented by the dashed curve. Also shown as a dot-dashed line are the median $v \sin i$ values for various spectral type bins. Near M4, the median rotational velocity appears to rise slightly, plateauing near $\sim 18 \mathrm{~km} \mathrm{~s}^{-1}$ well into the substellar mass regime. 


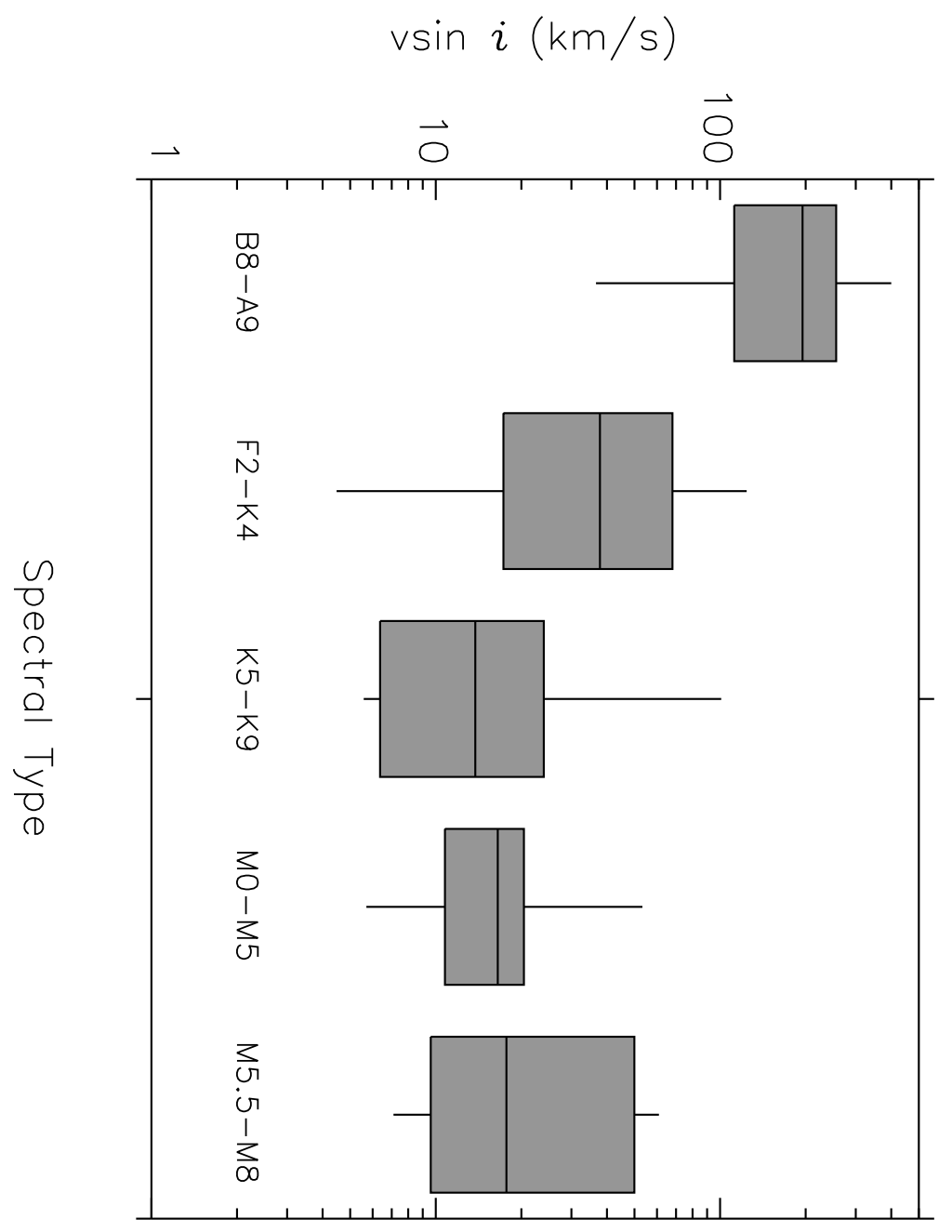

Fig. 5.- Boxplots of $v \sin i$ for the Upper Scorpius sample grouped into bins for B8-A9, F2K4, K5-K9, M0-M5, and M5.5-M8 spectral types. The central rectangles represent the first and third quartiles of each bin and the "whiskers" show the spread between minimum and maximum values. The horizontal lines within each box represent the median values for the spectral type bins. The median $v \sin i$ are: $195( \pm 70), 37.8( \pm 7.4), 13.8(+21.3,-8.2), 16.52$ $( \pm 5.3)$, and $17.72( \pm 8.1) \mathrm{km} \mathrm{s}^{-1}$ for the B8-A9, F2-K4, K5-K9, M0-M5, and M5.5-M8 spectral type bins, respectively. 

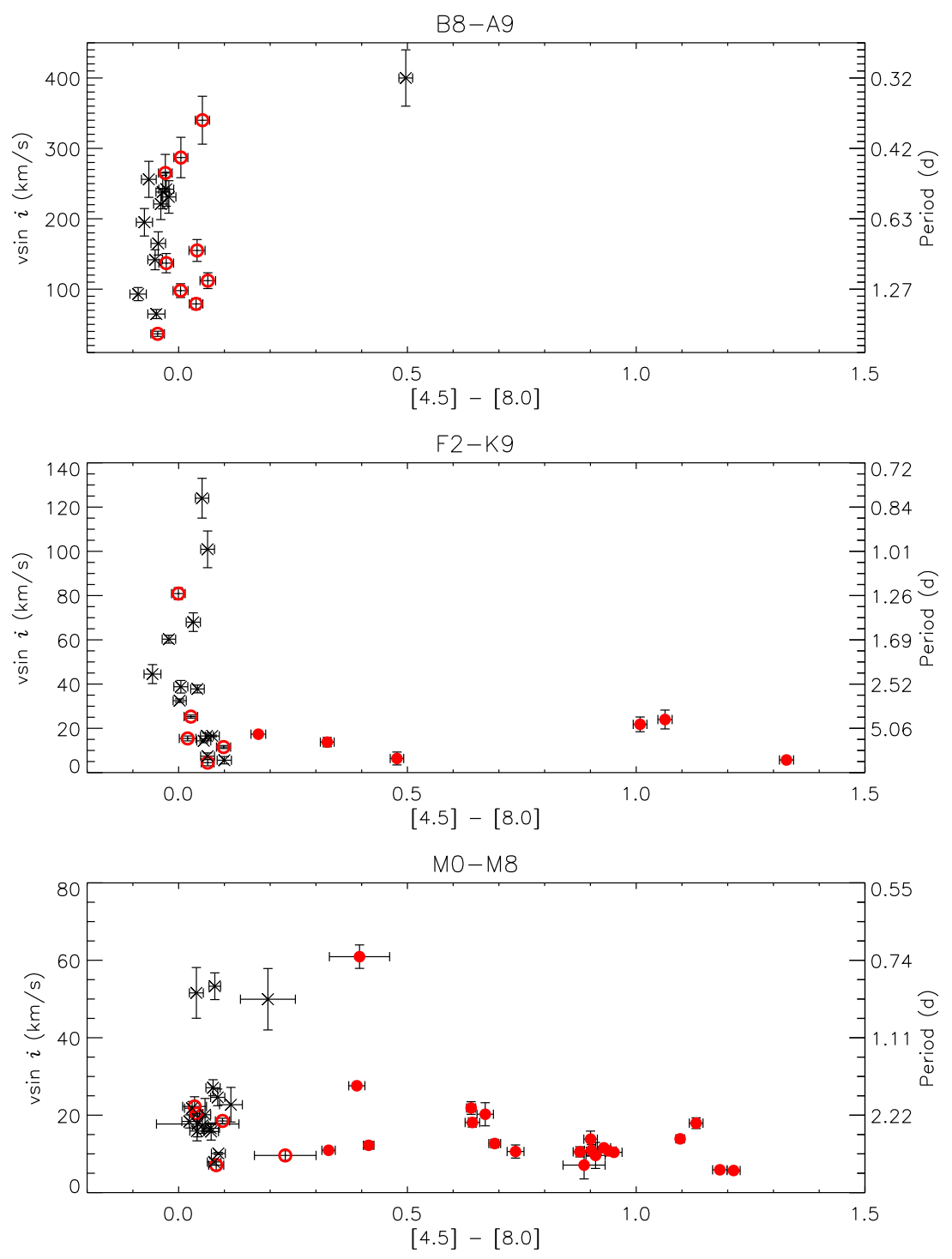

Fig. 6.- a) Projected rotational velocity, $v \sin i$, as a function of [4.5]-[8.0] color for B8-A9 (top panel), F2-K9 (middle panel), and M0-M8 (bottom panel) sources in Upper Scorpius. Symbols are as in Figure 4. To facilitate comparison with rotation period surveys, the mean velocities for fiducial rotation periods are plotted on the right ordinate, assuming nominal stellar radii for each spectral type or mass bin $\left(2.5 \mathrm{R}_{\odot}, 2.0 \mathrm{R}_{\odot}\right.$, and $0.88 \mathrm{R}_{\odot}$, for B8-A9, F2-K9, and M0-M8 type sources, respectively). Among the early-type (B8-A9) stars, the rotational velocities of the debris-disk and non-disk populations are indistinguishable. Considering the late-type (F2-M8) stars and brown dwarfs, however, we find that the disk-bearing sources are in general displaced toward lower $v \sin i$ relative to their non-excess counterparts. There also appear to be few slowly rotating, non-excess sources relative to younger star forming regions. 

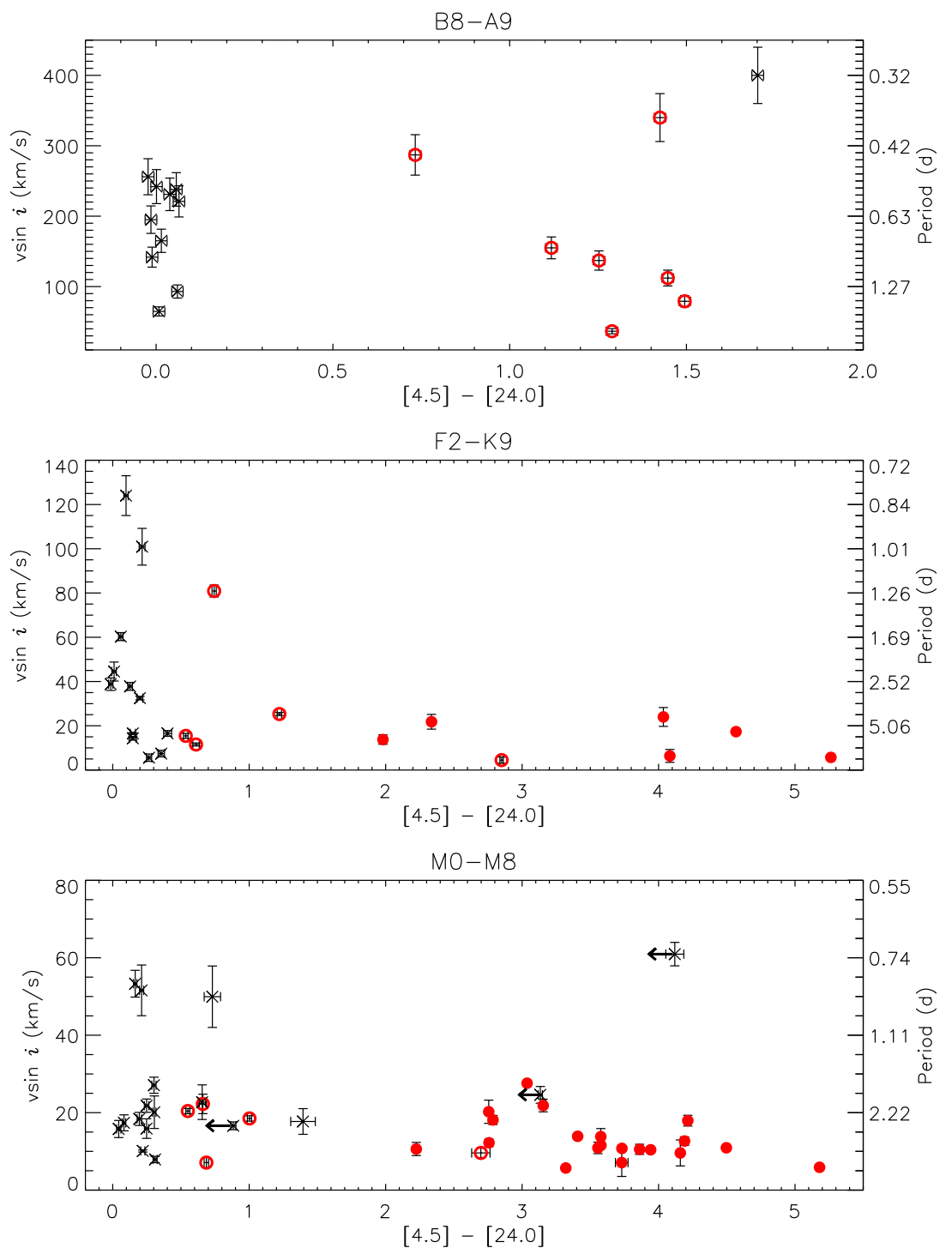

Fig. 6. - b) Projected rotational velocity, vsini, as a function of [4.5]-[24.0] color for B8-A9 (top panel), F2-K9 (middle panel), and M0-M8 (bottom panel) sources in Upper Scorpius. Symbols are as in Figure 4. The MIPS $24 \mu \mathrm{m}$ photometry for J161052.4-193734 is contaminated by bright nebulosity and that for ScoPMS 17 by a nearby star. Their [4.5]-[24.0] colors are represented by upper limits. SCH16235158-23172740 was not detected by MIPS and is also represented by a $3 \sigma$ upper limit. 

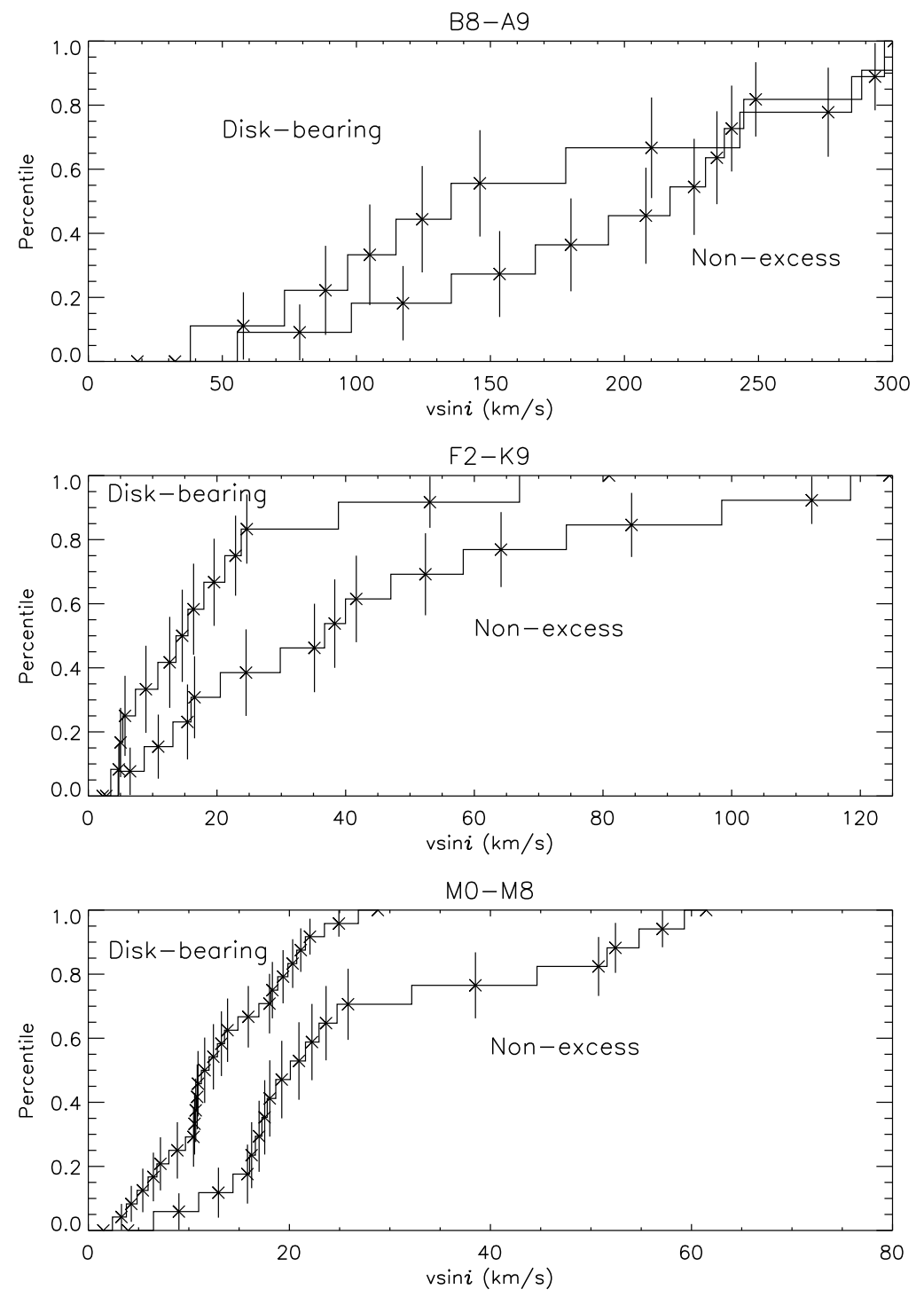

Fig. 7.- The cumulative distribution functions (CDF) for the disk-bearing and non-excess stars and brown dwarfs in Upper Scorpius, by spectral type or mass bin: B8-A9 (top panel), F2-K9 (middle panel), and M0-M8 (lower panel). The CDFs for the late-type, disk-bearing stars and brown dwarfs are substantially different from their non-disk counterparts, such that disk-bearing sources are rotating more slowly on average. 


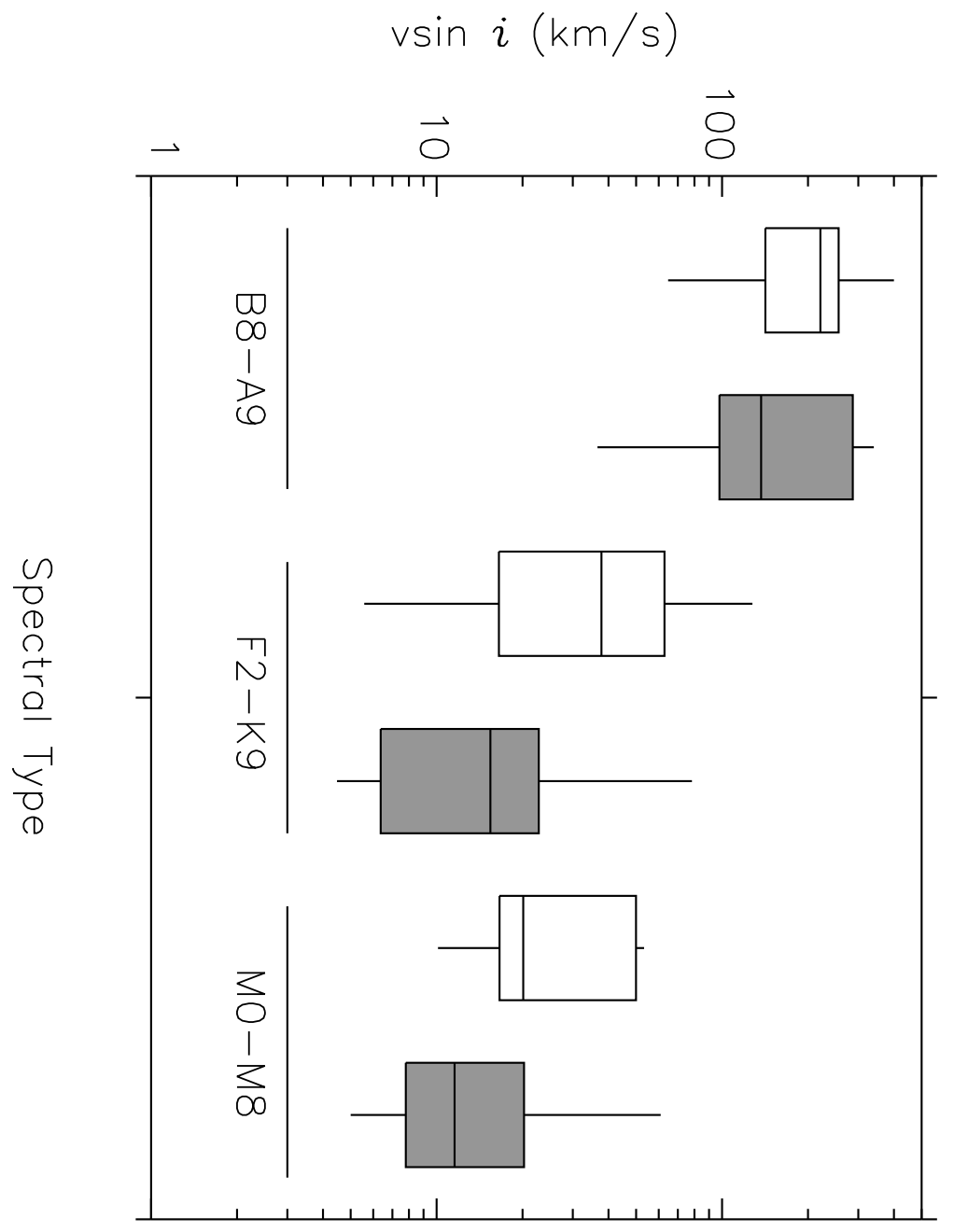

Fig. 8.- Boxplots of projected rotational velocities for disk-bearing and non-excess sources in Upper Scorpius, grouped by spectral type. Shaded boxes represent stars with primordial or debris disks, while clear boxes denote non-excess sources. The central rectangles represent the first and third quartiles of each bin and the "whiskers" span the range of $v \sin i$ values. The median of the distributions are marked by the solid horizontal line within each box. We find statistically significant differences between the rotational velocities for the disk-bearing and non-excess M-dwarfs in the association. 

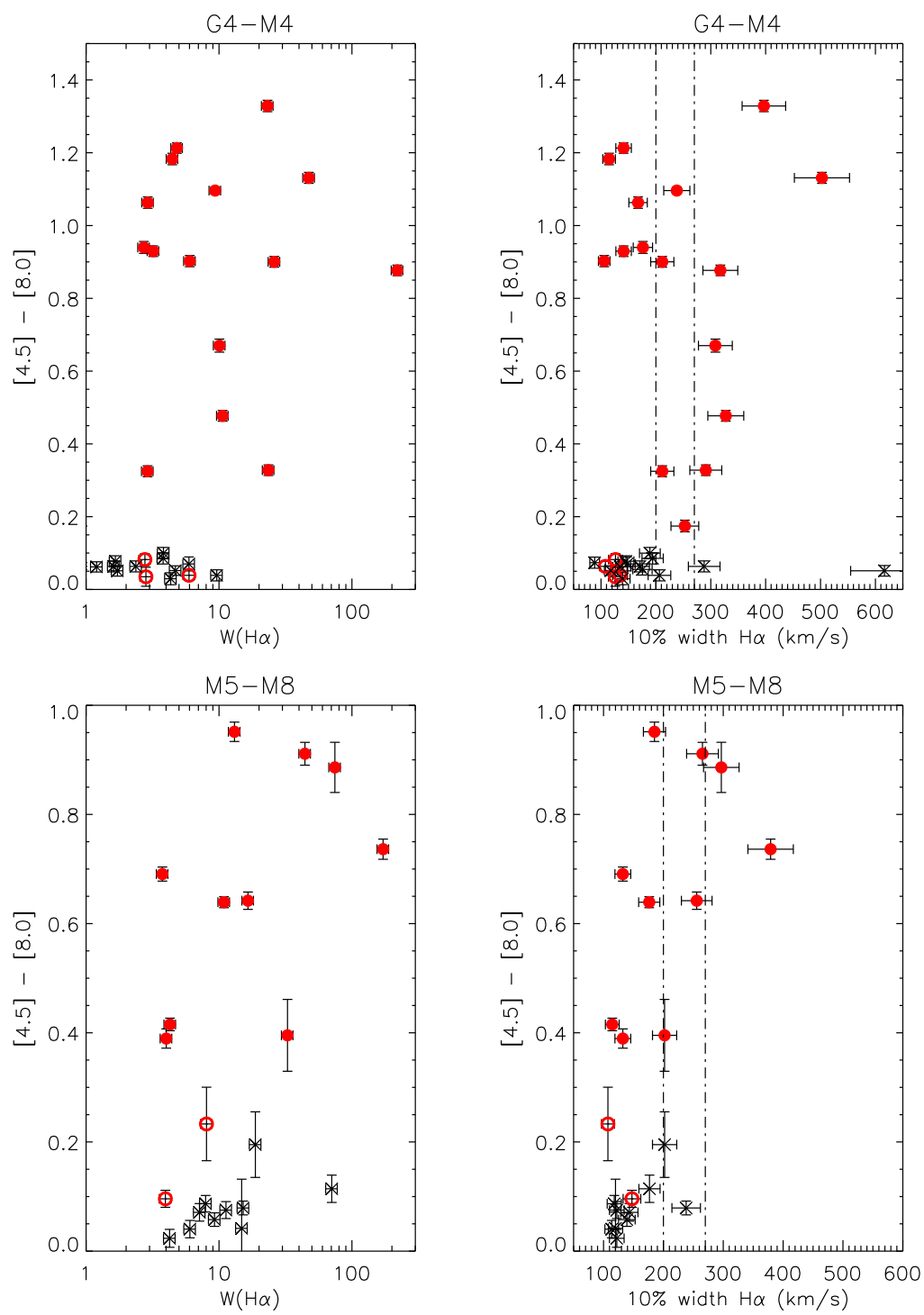

Fig. 9.- Spitzer IRAC [4.5]- [8.0] color plotted as a function of $\mathrm{W}(\mathrm{H} \alpha)$ (left panels) and $\mathrm{H} \alpha 10 \%$ velocity width (right panels) for the G4-M4 type stars in the Upper Scorpius spectroscopic sample (upper panels) and M5-M8 type sources (lower panels). Symbols are as in Figure 4. The vertical dashed lines in the $\mathrm{H} \alpha$ velocity width panels represent the 200 and $270 \mathrm{~km} \mathrm{~s}^{-1}$ velocity widths associated with accretion from Jayawardhana et al. (2003) for sources M5 and later and White and Basri (2003) for earlier spectral types. The errors shown represent the $1 \sigma$ photometric uncertainties and an assumed uncertainty of $10 \%$ for the measured $\mathrm{W}(\mathrm{H} \alpha)$ values and for the $\mathrm{H} \alpha$ velocity widths. The G4-type, non-excess source HD 142987 (H $\alpha 10 \%$ velocity width $\sim 600 \mathrm{~km} \mathrm{~s}^{-1}$ is a rapid rotator and possible spectroscopic binary. 

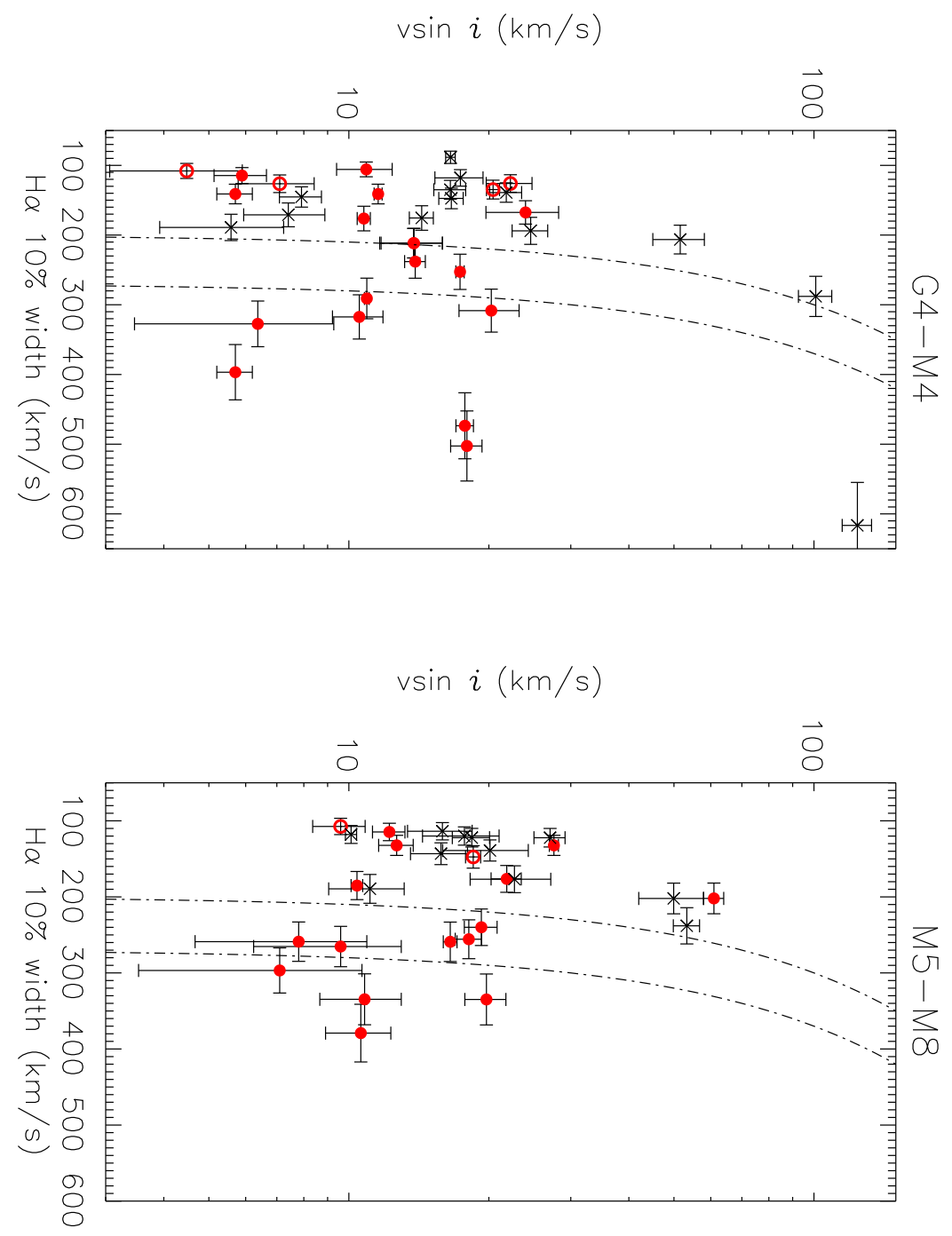

Fig. 10.- Projected rotational velocity plotted as a function of $\mathrm{H} \alpha 10 \%$ velocity width for the G4-M4 type stars in the Upper Scorpius sample (left panel) and for M5-M8 type sources (right panel). The curved dashed lines represent the intrinsic contributions of rotational line broadening to the $200 \mathrm{~km} \mathrm{~s}^{-1}$ and the $270 \mathrm{~km} \mathrm{~s}^{-1}$ velocity width criteria for accretion from Jayawardhana et al. (2003) and White and Basri (2003), respectively. 

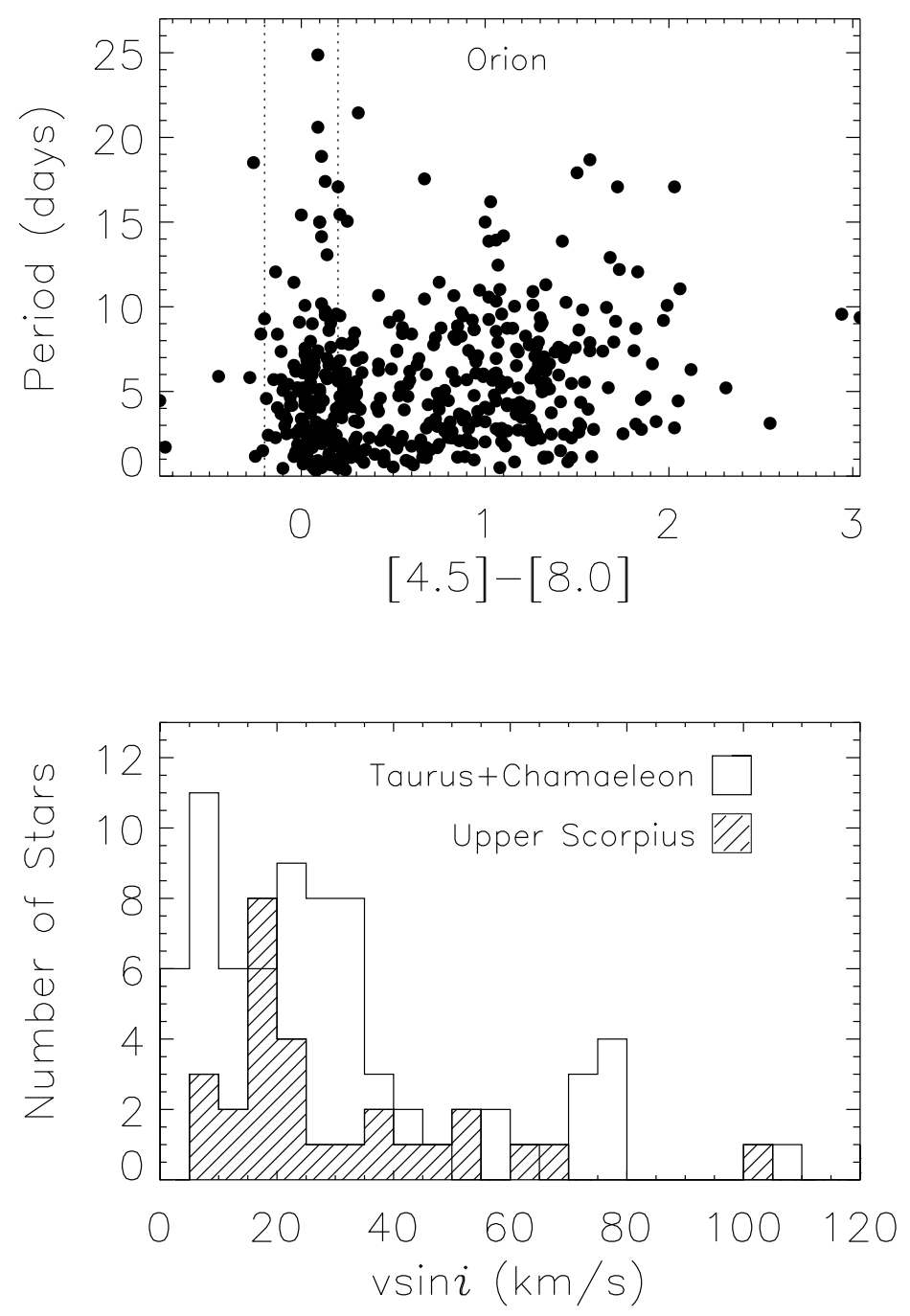

Fig. 11.- (upper panel) Rotation periods for 464 sources in Orion from Rebull et al. (2006) plotted as a function of [4.5]-[8.0] color. The vertical dot-dashed lines represent the adopted color boundaries for non-excess (i.e. non-disk bearing) stars. The fraction of slowly-rotating, non-excess sources in Orion having rotation periods of $>5$ days (corresponding to equatorial rotation velocities of $\leq 15 \mathrm{~km} \mathrm{~s}^{-1}$ for an assumed $1.5 R_{\odot}$ star) is $\sim 45 \%(63 / 140)$. (lower panel) Histograms of measured $v \sin i$ for late-type, non-excess sources in Upper Scorpius (cross-hatched region) and Taurus-Auriga + Chamaeleon I (from Nguyen et al. 2009). The fraction of slowly rotating ( $v \sin i \lesssim 15 \mathrm{~km} \mathrm{~s}^{-1}$ ), non-disk bearing sources in Upper Scorpius is $\sim 17 \%$ and that of Taurus and Chamaeleon I is $\sim 32 \%$. 
Table 1. Properties of the Upper Scorpius Spectroscopic Sample

\begin{tabular}{|c|c|c|c|c|c|c|c|c|c|c|c|}
\hline Source & Type & $\begin{array}{c}V_{R}^{\mathrm{a}} \\
\mathrm{km} \mathrm{s}^{-1}\end{array}$ & $\begin{array}{l}{[4.5]^{\mathrm{b}}} \\
\mathrm{mJy}\end{array}$ & $\begin{array}{l}{[8.0]^{\mathrm{b}}} \\
\mathrm{mJy}\end{array}$ & $\begin{array}{l}{[24]^{\mathrm{b}}} \\
\mathrm{mJy}\end{array}$ & $\begin{array}{c}v \sin i^{\mathrm{c}} \\
\mathrm{km} \mathrm{s}^{-1}\end{array}$ & $\begin{array}{c}\mathrm{W}(\mathrm{H} \alpha)^{\mathrm{d}} \\
\AA\end{array}$ & $\begin{array}{c}\mathrm{H} \alpha 10 \%^{\mathrm{e}} \\
\mathrm{km} \mathrm{s}^{-1}\end{array}$ & Disk Status & Epoch $^{f}$ & Comments \\
\hline HIP 78207 & B8 & $+1.54 \pm 2.23$ & 4019.5 & 2268.5 & 766.4 & $400:, 38.2 \pm 2.0^{\mathrm{i}}$ & -24.80 & $\ldots$ & Non-excess & H20070525 & $\mathrm{Be}, \mathrm{SB} 2$ \\
\hline HIP 79739 & B8 & $+14.58 \pm 1.58$ & 291.40 & 99.30 & 11.47 & $141.8 \pm 4.8$ & abs & $\ldots$ & Non-excess & M20080514 & Binaryg \\
\hline HIP 76071 & B9 & $-6.14 \pm 2.28$ & 277.00 & 93.20 & 10.79 & $256 \pm 10$ & abs & $\ldots$ & Non-excess & M20080514 & Binaryg \\
\hline HIP 77911 & B9 & $-2.90 \pm 2.56$ & 379.80 & 132.10 & 142.60 & $265 \pm 24$ & abs & $\ldots$ & Debris & H20070525 & Binary ${ }^{g}$ \\
\hline HIP 78809 & B9 & $-5.54 \pm 3.16$ & 213.10 & 72.80 & 8.54 & $64.7 \pm 1.7$ & $a b s$ & $\ldots$ & Non-excess & M20080514 & \\
\hline HIP 79410 & B9 & $-6.15 \pm 2.57$ & 279.10 & 104.60 & 41.25 & $340 \pm 19$ & abs & $\ldots$ & Debris & $\mathrm{H} 20070524$ & \\
\hline HIP 79439 & B9 & $-5.56 \pm 0.21$ & 305.40 & 109.60 & 23.86 & $287 \pm 26$ & abs & $\ldots$ & Debris & H20070524 & \\
\hline HIP 79785 & B9 & $-6.37 \pm 0.20$ & 443.90 & 153.60 & 18.61 & $238 \pm 13$ & abs & $\ldots$ & Non-excess & M20080514 & \\
\hline HIP 80024 & B9 & $-3.96 \pm 1.41$ & 368.20 & 126.10 & 48.03 & $36.6 \pm 1.5$ & abs & $\ldots$ & Debris & $\mathrm{H} 20070524$ & \\
\hline HIP 80493 & B9 & $-7.43 \pm 1.92$ & 285.20 & 97.80 & 11.50 & $165.1 \pm 6.2$ & abs & $\ldots$ & Non-excess & M20080514 & \\
\hline HIP 76310 & A0 & $-2.54 \pm 0.60$ & 247.60 & 88.80 & 165.40 & $97.9 \pm 2.2$ & abs & $\ldots$ & Debris & H20070524 & \\
\hline HIP 78847 & A0 & $-23.00 \pm 3.0$ & 261.70 & 91.20 & 10.42 & $242 \pm 14$ & abs & $\ldots$ & Non-excess & M20080514 & \\
\hline HIP 79124 & A0 & $-18.14 \pm 1.89$ & 299.40 & 103.20 & 12.64 & $221 \pm 11$ & abs & $\ldots$ & Non-excess & M20080514 & Binaryg \\
\hline HIP 79156 & A0 & $-3.73 \pm 1.91$ & 203.10 & 75.30 & 22.62 & $155.2 \pm 5.0$ & abs & $\ldots$ & Debris & H20070524 & Binary ${ }^{g}$ \\
\hline HIP 79860 & A0 & $-16.85 \pm 1.83$ & 127.60 & 44.70 & 5.26 & $231 \pm 16$ & abs & $\ldots$ & Non-excess & M20080514 & \\
\hline HIP 79878 & A0 & $-3.43 \pm 0.57$ & 268.90 & 93.70 & 33.90 & $137 \pm 5$ & abs & $\ldots$ & Debris & H20070524 & \\
\hline HIP 78963 & A9 & $-50.69 \pm 1.66$ & 225.50 & 75.20 & 8.85 & $195 \pm 12$ & abs & $\ldots$ & Non-excess & M20080514 & \\
\hline HIP 78996 & A9 & $-7.88 \pm 2.00$ & 237.80 & 90.10 & 35.87 & $112.1 \pm 10.1$ & abs & $\ldots$ & Debris & H20070524 & \\
\hline HIP 80088 & A9 & $-7.52 \pm 2.22$ & 143.50 & 53.10 & 22.62 & $79.1 \pm 4.7$ & abs & $\ldots$ & Debris & H20070524 & \\
\hline HIP 80130 & A9 & $-50.92 \pm 1.29$ & 215.00 & 70.80 & 9.04 & $93.0 \pm 2.8$ & abs & $\ldots$ & Non-excess & M20080514 & \\
\hline HIP 79643 & F2 & $-1.97 \pm 3.08$ & 117.90 & 42.10 & 9.30 & $80.9 \pm 2.7$ & abs & $\ldots$ & Debris & H20110424 & \\
\hline HIP 82319 & F3 & $-18.27 \pm 1.28$ & 133.30 & 46.70 & 5.60 & $60.3 \pm 1.8$ & abs & $\ldots$ & Non-excess & M20080514 & \\
\hline HIP 80896 & F3 & $\begin{array}{l}+3.33 \pm 2.67 \\
+\end{array}$ & 201.50 & 68.30 & 8.09 & $44.5 \pm 4.3$ & abs & $\ldots$ & Non-excess & M20080514 & Binaryg \\
\hline RXJ1550.9-2534 & F9 & $-6.02 \pm 1.56$ & 127.90 & 45.90 & 5.02 & $38.8 \pm 2.8$ & abs & $\ldots$ & Non-excess & M20080514 & \\
\hline [PZ99]J160000.7-250941 & G0 & $-1.33 \pm 0.30$ & 56.70 & 20.30 & 2.71 & $32.5 \pm 0.8$ & abs & $\ldots$ & Non-excess & H20110424 & \\
\hline [PZ99]J155812.7-232835 & $\mathrm{G} 2$ & $-4.56 \pm 0.21$ & 117.70 & 43.10 & 14.42 & $25.3 \pm 0.7$ & abs & $\ldots$ & Debris & H20110319 & \\
\hline HIP 79462 & G2 & $-4.99 \pm 1.16^{\mathrm{h}}$ & 232.00 & 84.40 & 15.11 & $15.43 \pm 1.11$ & abs & $\ldots$ & Debris & H20110424 & $\mathrm{SB} 2$ \\
\hline HD 142361 & $\mathrm{G} 3 \mathrm{~V}$ & $-5.84 \pm 1.62$ & 292.70 & 107.70 & 3.52 & $68.0 \pm 4.2$ & abs & & Non-excess & H20110424 & \\
\hline HD 142987 & G4 & $-6.43 \pm 2.09$ & 181.30 & 67.90 & 7.89 & $124.0 \pm 9.0$ & -1.72 & 616.5 & Non-excess & H20110424 & Rapid rotator \\
\hline RXJ16036-2245 & $\begin{array}{l}\text { G9 } \\
\text { G9 }\end{array}$ & $-10.41 \pm 0.18$ & 89.20 & 33.10 & $\begin{array}{l}3.09 \\
3.99\end{array}$ & $37.8 \pm 1.8$ & abs & $\ldots$ & Non-excess & M20080514 & \\
\hline [PZ99]J161411.0-230536 & Ko & $-6.31 \pm 1.41$ & 401.90 & 363.50 & 137.49 & $21.8 \pm 3.3$ & abs & $\ldots$ & Primordial & H20060616 & sub-arcsec binary \\
\hline [PZ99]J160421.7-213028 & K2 & $-6.90 \pm 0.24$ & 62.70 & 26.30 & 167.50 & $17.3 \pm 0.4$ & -0.27 & 252.7 & Primordial & H20060616 & Possible accretor \\
\hline [PZ99]J155847.8-175800 & K3 & $-7.17 \pm 0.10$ & 93.20 & 35.30 & 2.18 & $4.48 \pm 1.42$ & -0.76 & 108.0 & Debris & H20110319 & \\
\hline [PZ99]J160251.2-240156 & $\begin{array}{l}\text { no } \\
\text { K4 }\end{array}$ & $-6.53 \pm 0.20$ & 50.50 & $\begin{array}{l}03.00 \\
19.10\end{array}$ & $\begin{array}{l}2.10 \\
2.91\end{array}$ & $\begin{array}{l}4.40 \pm 1.42 \\
16.5 \pm 1.3\end{array}$ & $\begin{array}{l}-0.00 \\
-1.20\end{array}$ & 135.0 & Non-excess & H20110424 & \\
\hline ScoPMS 45 & K5 IV & $-7.86 \pm 0.10$ & 78.50 & 30.70 & 5.48 & $11.54 \pm 0.70$ & abs & $\ldots$ & Debris & H20110424 & \\
\hline [PZ99]J160357.6-203105 & K5 & $-6.66 \pm 0.88$ & 191.90 & 106.40 & 328.00 & $6.37 \pm 2.91$ & -10.65 & 327.38 & Primordial & $\mathrm{H} 20060616$ & Accretor \\
\hline [PZ99]J160856.7-203346 & K5 & $-9.18 \pm 0.20$ & 73.50 & 28.10 & 3.35 & $16.54 \pm 0.39$ & -0.10 & 88.46 & Non-excess & M20080514 & \\
\hline J160643.8-190805 & K6 & $-5.43 \pm 0.56$ & 52.30 & 25.20 & 12.89 & $13.77 \pm 2.12$ & -2.90 & 211.61 & Primordial & H20060616 & sub-arcsec binary \\
\hline [PZ99]J160042.8-212737 & K7 & $\begin{array}{l}+1.99 \pm 7.08 \\
+1.08\end{array}$ & 53.60 & 20.30 & 2.60 & $100.9 \pm 8.3$ & -2.37 & 287.98 & Non-excess & H20110424 & Rapid rotator \\
\hline [PZ99]J160239.1-254208 & K7 & $-3.60 \pm 0.15$ & 46.30 & 17.40 & 2.11 & $14.34 \pm 0.85$ & -0.74 & 175.50 & Non-excess & H20110424 & \\
\hline J161031.9-191305 & $\begin{array}{l}\text { Ki } \\
\text { K7 }\end{array}$ & $\begin{array}{l}-3.00 \pm 0.10 \\
-6.91 \pm 0.27\end{array}$ & 60.00 & 23.50 & 3.05 & $\begin{array}{l}14.04 \pm 0.00 \\
5.58 \pm 1.66\end{array}$ & $\begin{array}{l}-0.4 \\
-3.82\end{array}$ & $\begin{array}{l}18.00 \\
189.02\end{array}$ & $\begin{array}{l}\text { Non-excess } \\
\text { Nonces }\end{array}$ & H20110424 & \\
\hline $\mathrm{J} 160801.4-202741$ & K8 & $-7.08 \pm 0.36$ & 40.40 & 15.30 & 2.23 & $7.41 \pm 1.47$ & -1.61 & 170.99 & Non-excess & H20110424 & \\
\hline $\mathrm{J} 160823.2-193001$ & K9 & $+6.18 \pm 2.68$ & 47.00 & 44.70 & 76.90 & $23.98 \pm 4.27$ & -2.91 & 167.51 & Primordial & $\mathrm{H} 20060616$ & SB2(?) \\
\hline $\mathrm{J} 160900.7-190852$ & K9 & $-7.30 \pm 0.23$ & 56.40 & 68.50 & 285.80 & $\leq 5.7$ & -23.17 & 396.75 & Primordial & H20060616 & Accretor \\
\hline [PZ99]J160831.4-180241 & M0 & $-9.73 \pm 0.12$ & 54.20 & 20.80 & 2.87 & $7.91 \pm 0.82$ & -1.66 & 145.34 & Non-excess & M20080514 & \\
\hline J161420.2-190648 & Mo & $-6.77 \pm 1.77$ & 606.50 & 614.10 & 1170.00 & $17.94 \pm 1.39$ & -47.42 & 502.56 & Primordial & H20060616 & Accretor \\
\hline ScoPMS 31 & M0.5 & $-4.53 \pm 0.27$ & 133.70 & 64.60 & 334.60 & $10.93 \pm 0.13$ & -23.53 & 290.93 & Primordial & H20060616 & Accretor, sub-arcsec binary \\
\hline ScoPMS 17 & M1 & $-3.70 \pm 0.75$ & 60.80 & 23.50 & 43.36 & $24.60 \pm 2.16$ & -3.80 & 193.97 & Non-excess & H20060616 & \\
\hline J160954.4-190654 & M1 & $-6.87 \pm 0.23$ & 31.40 & 12.10 & 2.35 & $\leq 7.1$ & -2.77 & 126.38 & Debris & M20080513 & \\
\hline
\end{tabular}


Table 1-Continued

\begin{tabular}{|c|c|c|c|c|c|c|c|c|c|c|c|}
\hline Source & Type & $\begin{array}{c}V_{R}^{\mathrm{a}} \\
\mathrm{km} \mathrm{s}^{-1}\end{array}$ & $\begin{array}{l}{[4.5]^{\mathrm{b}}} \\
\mathrm{mJy}\end{array}$ & $\begin{array}{l}{[8.0]^{\mathrm{b}}} \\
\mathrm{mJy}\end{array}$ & $\begin{array}{l}{[24]^{\mathrm{b}}} \\
\mathrm{mJy}\end{array}$ & $\begin{array}{c}v \sin i^{\mathrm{c}} \\
\mathrm{km} \mathrm{s}^{-1}\end{array}$ & $\underset{\AA}{\mathrm{W}(\mathrm{H} \alpha)^{\mathrm{d}}}$ & $\begin{array}{c}\mathrm{H} \alpha 10 \% \\
\mathrm{~km} \mathrm{~s}^{-1}\end{array}$ & Disk Status & $\operatorname{Epoch}^{f}$ & Comments \\
\hline J161115.3-175721 & M1 & $-7.23 \pm 0.17$ & 86.00 & 93.90 & 72.82 & $\leq 5.7$ & -4.82 & 141.08 & Primordial & H20060616 & \\
\hline J160341.8-200557 & M2 & $-3.71 \pm 1.00^{\mathrm{h}}$ & 35.00 & 13.00 & 1.76 & & -1.70 & 198.00 & Non-excess & M20080513 & $\mathrm{SB} 2$ \\
\hline J160357.9-194210 & M2 & $-3.35 \pm 0.16$ & 23.90 & 20.10 & 25.65 & $11.56 \pm 0.23$ & -3.20 & 141.10 & Primordial & $\mathrm{H} 20060616$ & \\
\hline J160545.4-202308 & M2 & & 21.20 & 18.00 & 26.22 & $10.77 \pm 0.35$ & -2.72 & 176.30 & Primordial & H20060616 & SB2, Visual binary \\
\hline J155829.8-231007 & M3 & $-4.37 \pm 3.03$ & 17.60 & 14.10 & 24.53 & $10.53 \pm 1.31$ & -219.51 & 317.41 & Primordial & $\mathrm{H} 20070524$ & Accretor \\
\hline J160953.6-175446 & M3 & $-5.34 \pm 1.28$ & 8.55 & 7.00 & 9.17 & $13.81 \pm 2.08$ & -26.03 & 211.61 & Primordial & $\mathrm{H} 20070524$ & \\
\hline J161052.4-193734 & M3 & $-7.94 \pm 1.36$ & 12.70 & 4.84 & 1.14 & $16.61 \pm 1.00$ & -5.94 & 147.44 & Non-excess & M20080513 & \\
\hline J155624.8-222555 & M4 & $-6.32 \pm 0.86$ & 22.80 & 18.70 & 23.98 & $10.90 \pm 1.49$ & -6.02 & 105.80 & Primordial & H20070524 & \\
\hline J155706.4-220606 & M4 & $-6.02 \pm 1.80$ & 15.10 & 10.00 & 7.60 & $20.24 \pm 2.99$ & -10.08 & 308.38 & Primordial & H20070525 & Accretor \\
\hline J155729.9-225843 & M4 & $-1.06 \pm 1.03$ & 6.65 & 6.52 & 6.10 & $13.89 \pm 0.71$ & -9.35 & 238.07 & Primordial & H20070525 & \\
\hline J155918.4-221042 & M4 & $-7.93 \pm 0.77$ & 24.20 & 8.96 & 1.17 & $51.58 \pm 6.55$ & -9.62 & 206.41 & Non-excess & M20080513 & SB2(?) \\
\hline J160439.1-194245 & M4 & $-3.67 \pm 2.26$ & 11.90 & 4.39 & 0.87 & $22.25 \pm 2.51$ & -2.82 & 126.0 & Debris & H20110319 & \\
\hline $\mathrm{J} 160708.7-192733$ & M4 & $-1.97 \pm 0.91$ & 8.48 & 3.14 & 0.56 & $20.42 \pm 0.66$ & -5.95 & 134.77 & Debris & M20080516 & \\
\hline J160801.5 - 192733 & M4 & $-4.44 \pm 0.50$ & 31.60 & 11.60 & 1.58 & $21.80 \pm 1.72$ & -4.33 & 139.00 & Non-excess & M20080515 & \\
\hline J160959.4-180009 & M4 & $-6.28 \pm 0.80$ & 24.20 & 25.70 & 113.60 & $5.89 \pm 0.76$ & -4.45 & 114.63 & Primordial & H20070525 & \\
\hline J161026.4-193950 & M4 & $-7.43 \pm 1.69$ & 19.80 & 7.42 & 0.85 & $17.37 \pm 2.06$ & -4.70 & 117.97 & Non-excess & M20080515 & \\
\hline SCH16014156-21113855 & M4 & $-12.81 \pm 1.00$ & & & & $17.76 \pm 0.77$ & -95.95 & 473.57 & & M20080516 & Accretor \\
\hline $\mathrm{J} 160159.7-195219$ & M5 & $-5.32 \pm 1.57$ & 6.77 & 2.62 & 0.33 & $10.11 \pm 0.28$ & -7.93 & 117.93 & Non-excess & M20080513 & \\
\hline J160210.9-200749 & M5 & $-4.28 \pm 0.76$ & 7.00 & 2.67 & 0.29 & $15.78 \pm 2.21$ & -7.13 & 143.22 & Non-excess & M20080515 & \\
\hline J160449.9-203835 & M5 & $-3.06 \pm 2.08$ & 11.20 & 4.22 & 0.59 & $20.11 \pm 4.20$ & -9.25 & 138.99 & Non-excess & M20080515 & \\
\hline J160525.5-203539 & M5 & $-3.26 \pm 1.75$ & 11.80 & 6.18 & 5.95 & $12.22 \pm 0.98$ & -4.28 & 114.63 & Primordial & H20070525 & \\
\hline J160531.5 - 192623 & M5 & $-5.22 \pm 1.17$ & 6.66 & 2.55 & 0.35 & $27.08 \pm 2.08$ & -11.28 & 122.19 & Non-excess & M20080513 & \\
\hline J160532.1-193315 & M5 & $-3.36 \pm 1.58$ & 12.70 & 8.94 & 3.92 & $10.61 \pm 1.70$ & -171.67 & 379.12 & Primordial & $\mathrm{H} 20070525$ & Accretor \\
\hline J160600.6-195711 & M5 & $-5.44 \pm 1.80$ & 21.90 & 11.20 & 14.28 & $27.60 \pm 0.62$ & -4.01 & 132.25 & Primordial & H20070524 & \\
\hline J160611.9-193532 & M5 & $-7.38 \pm 5.84$ & 10.80 & 4.15 & 0.50 & $53.30 \pm 3.45$ & -15.21 & 238.04 & Non-excess & $\mathrm{H} 20070525$ & Rapid rotator \\
\hline J160622.8-201124 & M5 & $-5.09 \pm 0.68$ & 10.40 & 7.02 & 19.63 & $12.67 \pm 1.08$ & -3.75 & 132.25 & Primordial & H20070524 & \\
\hline J160702.1-201938 & M5 & $-4.83 \pm 2.72$ & 11.90 & 7.66 & 8.65 & $21.85 \pm 1.63$ & -10.90 & 176.32 & Primordial & $\mathrm{H} 20070524$ & Visual binary \\
\hline $\mathrm{J} 160802.4-202233$ & M5 & $-5.84 \pm 1.30$ & 15.40 & 5.71 & 0.77 & $15.89 \pm 2.52$ & -6.04 & 113.71 & Non-excess & M20080515 & \\
\hline J160827.5-194904 & M5 & $-6.49 \pm 2.11$ & 17.20 & 11.10 & 8.91 & $18.11 \pm 1.18$ & -16.51 & 255.69 & Primordial & $\mathrm{H} 20070524$ & Accretor \\
\hline J160900.0-190836 & M5 & $-8.34 \pm 1.48$ & 14.10 & 12.10 & 21.17 & $10.41 \pm 0.30$ & -13.07 & 185.13 & Primordial & $\mathrm{H} 20070524$ & \\
\hline J160915.8-193706 & M5 & $-4.10 \pm 1.11$ & 6.93 & 2.53 & 0.33 & $18.38 \pm 1.69$ & -4.25 & 122.14 & Non-excess & M20080515 & \\
\hline J161011.0-194603 & M5 & $-8.52 \pm 0.87$ & 7.79 & 3.04 & 0.78 & $18.51 \pm 0.69$ & -3.95 & 147.44 & Debris & M20080515 & \\
\hline SCH16033470-18293060 & M5 & $-7.34 \pm 2.37$ & $\ldots$ & $\ldots$ & $\ldots$ & $19.76 \pm 2.00$ & -25.06 & 334.87 & $\ldots$ & M20080516 & Accretor \\
\hline SCH16081081-22294303 & M5 & $-10.93 \pm 2.05$ & $\ldots$ & $\ldots$ & $\ldots$ & $16.52 \pm 4.05$ & -30.54 & 259.05 & $\ldots$ & M20080516 & Accretor \\
\hline SCH16150524-24593500 & M5 & $-16.22 \pm 2.12$ & $\ldots$ & $\ldots$ & $\ldots$ & $7.80 \pm 3.13$ & -21.83 & 258.88 & $\ldots$ & M20080516 & Accretor \\
\hline SCH16092137-21393452 & M5.5 & $-12.63 \pm 0.3$ & $\cdots$ & $\ldots$ & $\ldots$ & $11.10 \pm 2.05$ & -22.79 & 189.44 & $\ldots$ & M20080516 & \\
\hline SCH16305349-24245439 & M5.5 & $-8.87 \pm 0.46$ & $\ldots$ & & & $19.27 \pm 1.56$ & -30.31 & 239.94 & & M20080516 & Accretor \\
\hline SCH16093018-20595409 & M6 & $-5.08 \pm 0.60$ & 1.94 & 0.859 & 0.93 & $\leq 9.6$ & -8.05 & 107.36 & Debris (?) & M20080513 & \\
\hline SCH16103876-18292353 & M6 & $-6.83 \pm 0.82$ & 3.70 & 2.99 & 4.57 & $\overline{\leq} 7.1$ & -74.44 & 296.75 & Primordial & M20080514 & Accretor \\
\hline SCH16200756-23591522 & M6 & $-10.71 \pm 5.06$ & 4.49 & 1.92 & 0.35 & $49.94 \pm 7.93$ & -18.78 & 202.06 & Non-excess & M20080513 & SB2(?) \\
\hline SCH16202127-21202923 & M6 & $-5.92 \pm 0.90$ & 3.34 & 1.24 & 0.48 & $17.72 \pm 3.32$ & -14.81 & 119.96 & Non-excess & M20080514 & \\
\hline SCH16263026-23365552 & M6 & $-12.15 \pm 1.20$ & 8.09 & 6.69 & 14.84 & $\leq 9.6$ & -44.31 & 265.28 & Primordial & M20080515 & Accretor \\
\hline SCH16284706-24281413 & M6 & $-10.56 \pm 0.50$ & & & & $10.81 \pm 2.15$ & -189.17 & 334.67 & & M20080516 & Accretor \\
\hline SCH16224384-19510575 & M8 & $-7.87 \pm 1.28$ & 11.64 & 4.62 & 0.85 & $22.70 \pm 4.47$ & -70.52 & 176.77 & Non-excess & M20080515 & SB2(?) \\
\hline SCH16235158-23172740 & M8 & $-7.47 \pm 2.45$ & 3.52 & 1.81 & $<6.22$ & $60.94 \pm 3.03$ & -32.75 & 202.02 & Primordial & M20080514 & Rapid rotator \\
\hline
\end{tabular}

${ }^{\mathrm{a}}$ Heliocentric radial velocity. 
${ }^{\mathrm{b}}$ IRAC and MIPS fuxes from Carpenter et al. (2006) and Carpenter et al. (2009), respectively.

${ }^{\mathrm{c}}$ Projected rotational velocities.

${ }^{d}$ Equivalent width of $\mathrm{H} \alpha$. Negative values imply emission.

${ }^{e}$ Velocity width of $\mathrm{H} \alpha$ emission profiles at $10 \%$ of peak flux.

${ }^{\mathrm{f}} \mathrm{UT}$ Date of observation (yyyymmdd) with prefix: H=HIRES, $\mathrm{M}=\mathrm{MIKE}$

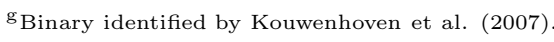

${ }^{\mathrm{h}}$ Systemmic velocity reported for double-line spectroscopic binaries.

${ }^{\mathrm{i}} v \sin i$ provided for both primary and secondary components. 
Table 2. Radial and Rotational Velocity Measurements from the Literature

\begin{tabular}{lcc}
\hline \hline \multicolumn{1}{c}{ Source } & $\begin{array}{c}V_{R}^{\mathrm{a}, b} \\
\left(\mathrm{~km} \mathrm{~s}^{-1}\right)\end{array}$ & $\begin{array}{c}v \sin i \mathrm{~b}, c \\
\left(\mathrm{~km} \mathrm{~s}^{-1}\right)\end{array}$ \\
\hline HIP 78207 & $-5.6(1)$ & $400(2)$ \\
HIP 79739 & $-14.7(3)$ & $160(2)$ \\
HIP 77911 & $+3(\mathrm{ref} 1) ;-7.4(3)$ & $300:(2)$ \\
HIP 79410 & $-9.4(3)$ & $180(2)$ \\
HIP 79439 & $-9.5(3)$ & $160:(2)$ \\
HIP 79785 & $-8(1) ;-10.7(3)$ & $300:(2)$ \\
HIP 80024 & $-9(4) ;-7.9(3)$ & $\leq 50(2)$ \\
HIP 76310 & $+4.4(3)$ & $\ldots$ \\
HD 142361 & $-3.39(5) ;-3.9(6)$ & $56(6)$ \\
[PZ99]J155812.7-232835 & $-7.0(6)$ & $\ldots$ \\
[PZ99] J161411.0-230536 & $-4.9(6)$ & $27.5(6)$ \\
RXJ16036-2245 & $-5.7(6)$ & $34.0(6)$ \\
ScoPMS 45 & $-7.7(7)$ & $14(7)$ \\
ScoPMS 31 & $-3.7(7)$ & $<15(7)$ \\
ScoPMS 17 & $-3.5(7)$ & $35(7)$ \\
SCH16224384-19510575 & $-10.2(8)$ & $25(8)$ \\
SCH16235158-23172740 & $-6.0(8)$ & $53(8)$ \\
\hline
\end{tabular}

${ }^{\mathrm{a}}$ Heliocentric radial velocity.

${ }^{b}$ References: 1) Evans (1967); 2) Slettebak (1968); 3) Sartori et

al. (2003); 4) Wilson (1953); 5) White et al. (2007); 6) Torres et

(1994); 8) Rice et al. (2008).

${ }^{\mathrm{c}}$ Projected rotational velocity. 